\title{
Three-Dimensional Microwave Holographic Imaging Employing Forward-Scattered Waves Only
}

\author{
Reza K. Amineh,, ${ }^{1,2}$ Maryam Ravan, ${ }^{1,2}$ Justin McCombe, ${ }^{1}$ and Natalia K. Nikolova ${ }^{1}$ \\ ${ }^{1}$ Department of Electrical and Computer Engineering, McMaster University, Hamilton, Canada L8S 4 K1 \\ ${ }^{2}$ Department of Electrical and Computer Engineering, University of Toronto, Toronto, Canada M5S $3 G 4$ \\ Correspondence should be addressed to Reza K. Amineh; khalajr@mcmaster.ca
}

Received 24 April 2013; Accepted 8 July 2013

Academic Editor: Francesco Soldovieri

Copyright (c) 2013 Reza K. Amineh et al. This is an open access article distributed under the Creative Commons Attribution License, which permits unrestricted use, distribution, and reproduction in any medium, provided the original work is properly cited.

\begin{abstract}
We propose a three-dimensional microwave holographic imaging method based on the forward-scattered waves only. In the proposed method, one transmitter and multiple receivers perform together a two-dimensional scan on two planar apertures on opposite sides of the inspected domain. The ability to achieve three-dimensional imaging without back-scattered waves enables the imaging of high-loss objects, for example, tissues, where the back-scattered waves may not be available due to low signal-to-noise ratio or nonreciprocal measurement setup. The simulation and experimental results demonstrate the satisfactory performance of the proposed method in providing three-dimensional images. Resolution limits are derived and confirmed with simulation examples.
\end{abstract}

\section{Introduction}

Better penetration of microwaves inside lossy dielectric objects, compared to visible light, provides a means for inspection of biological tissues, wood, concrete, ceramics, soil, and so forth. Various techniques have been proposed to harness the ability of microwaves for two-dimensional (2D) and three-dimensional (3D) imaging in a wide range of applications such as biomedical imaging [1], concealed weapon detection [2], through-the-wall imaging [3], and nondestructive testing and evaluation [4]. All these methods operate on the scattered signals at the antenna terminals but they differ significantly in the data acquisition schemes and the processing algorithms. A summary of major microwave imaging techniques is available in [5].

In this paper, the focus is on microwave holographic imaging. Microwave holography was originally developed by Farhat et al. $[2,6,7]$ in a form similar to acoustic holography $[8,9]$, which can be viewed as a long-wavelength implementation of the original optical holography developed by Gabor [10]. These microwave/acoustic holography techniques operate by recording a "hologram" which contains information about the magnitude and the phase of the wave reflected by a target. The hologram is captured on a film by interfering the scattered wave due to the examined object with a reference wave. This interference pattern or "hologram" can then be used to reconstruct an image of the object by illuminating the hologram with the same reference wave. In modern microwave/acoustic holography, scanned transceivers capture the scattered wavefront's phase and amplitude distributions directly. Once recorded, the image can be reconstructed digitally using a Fourier-optics image reconstruction that essentially emulates the hologram-based reconstruction process.

In [11], a 3D microwave holographic imaging technique has been proposed. The authors described the technique as the merging of the single-frequency $2 \mathrm{D}$ holographic imaging methods with wideband 2D synthetic aperture radar (SAR). The SAR imaging systems are commonly used to obtain high-resolution 2D images of a terrain at long ranges. These systems operate by scanning a wide bandwidth radar transceiver along a long linear aperture, typically using an airplane. Then they reconstruct a 2D image along the scanned line (e.g., see [12-15]). In [11], wideband back-scattered data is collected over a rectangular aperture. The system is quasi-monostatic that is, the transmitting and the receiving antennas are separate but colocated during the $2 \mathrm{D}$ scan. The processing relies on an assumed analytical (exponential) form of the incident field and Green's function in order to cast the inversion in the form of a 3D inverse Fourier transform (FT). 
This limits the technique to far-zone imaging. Resampling of the data in $k_{z}$-space is also necessary. Also, in [16-18], similar $3 \mathrm{D}$ holographic imaging techniques have been proposed where the wideband data is acquired on a cylindrical aperture to extend the "view angles" for the object. This leads to better imaging results.

In $[19,20]$, we extended the 3D holography technique in [11] to near-field imaging. The proposed method allows for incorporating forward-scattered signals in addition to the back-scattered signals. The method also allows for incidentfield and Green's function distributions in numeric forms. These are obtained through simulations of the particular background medium with the particular Tx/Rx antennas (Tx and $\mathrm{Rx}$ stand for transmitter and receiver, resp.). The accurate representations of the incident field and Green's function for the particular acquisition setup and antennas (not assuming a plane-wave exponential form as in [11]) are crucial in nearfield imaging. The procedure in [11] is inapplicable with numeric representations of the incident field and the Green's function. Thus, the numerical form of the incident field and the Green's function required a new inversion procedure. In $[19,20]$, a linear system of equations is solved for each spatial frequency pair $\left(k_{x}, k_{y}\right)$ and $2 \mathrm{D}$ inverse FT is applied to the solution on planes (slices) at all desired range locations. These linear systems of equations have much smaller dimensions and they are less ill-conditioned compared to the systems of equations in regular optimization-based microwave imaging techniques.

Also, the algorithm is not limited to propagating waves (where $k_{z}$ is assumed real as in [11]) and is thus capable of processing the evanescent waves available in near-field imaging. Processing larger values for $\left(k_{x}, k_{y}\right)$ leads to better spatial resolution of the reconstructed images. Furthermore, in the approach proposed in [11], resampling of the data in $k_{z^{-}}$ space is necessary which may lead to additional errors. Algorithms such as the Stolt interpolation [21] and nonuniform fast Fourier transform (NUFFT) [22] have been proposed to alleviate or avoid this problem. Even if the resampling (interpolation) errors can be alleviated, assuming $k_{x}, k_{y}$, and $k_{z}$ as independent variables still leads to errors in the image reconstruction process. These issues are irrelevant in our technique since the use of the parameter $k_{z}$ is avoided.

In [20], we presented experimental holographic imaging results when employing the TEM horn antennas proposed in [23]. Thus, the main focus was on applications for breastcancer diagnostics and artificial glycerin-based phantoms emulating the breast tissues were imaged. Only 2D holographic imaging results were presented employing forwardscattered waves. This is due to the fact that the back-scattered (or reflected) signals were measured through the complex reflection coefficient of the Tx antenna. Such signals are so weak that they are masked by the measurement noise and uncertainties. On the other hand, the back-scattered signals are crucial for implementing $3 \mathrm{D}$ holographic imaging with planar scanning.

In this paper, we propose a configuration which allows for $3 \mathrm{D}$ holographic imaging where only forward-scattered data is available. We develop expressions for the resolution limits of bistatic holography using an approach developed for

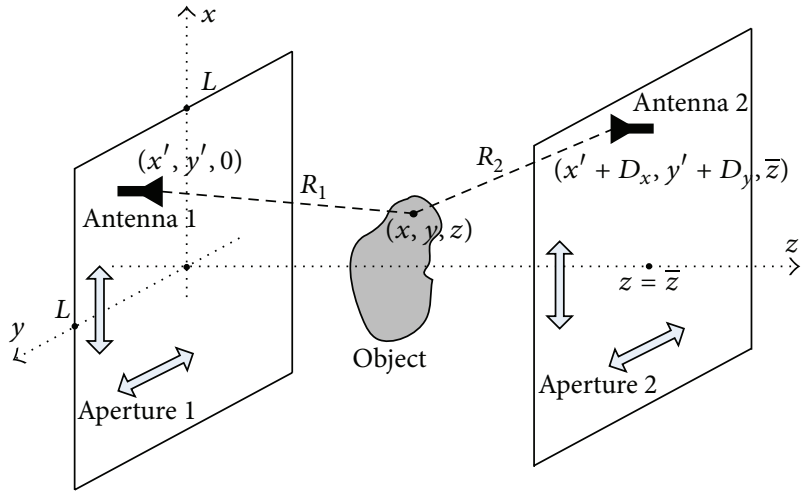

FIGURE 1: Microwave holography setup. The grey thick arrows show the directions of $2 \mathrm{D}$ scan.

bistatic SAR [24]. We confirm that the availability of forwardscattered data in a planar surface acquisitions similar to those in $[19,20]$ cannot provide enough information to perform $3 \mathrm{D}$ holographic imaging. This is consistent with our previous observations. The images obtained when we exclude the back-scattered data do not have depth resolution. Moreover, the image quality in the cross range is also compromised when attempting $3 \mathrm{D}$ reconstruction without back-scattered signals, although a $2 \mathrm{D}$ holographic reconstruction in the cross-range plane of the object is successful. Overall, without the back-scattered data, the holographic reconstructed images contain strong artifacts along both range and cross range. The solution to this problem as proposed in this work is a scanning setup comprising one transmitter and five receivers which move together during the $2 \mathrm{D}$ scan on two rectangular apertures on opposite sides of the inspected region. We show that $3 \mathrm{D}$ image reconstruction is possible with this setup when employing only forward-scattered data. We also derive the range and cross-range resolution limits.

\section{Resolution in Two-Antenna Configuration}

We consider a two-antenna microwave holography setup where the antennas scan simultaneously two rectangular planar surfaces in a raster pattern. This setup is illustrated in Figure 1 where antenna 1 and antenna 2 perform the scan together on aperture 1 at $z=0$ and aperture 2 at $z=\bar{z}$. At each sampling step, the wideband transmission coefficient of this two-port system is acquired, which represents the forward-scattered wave. We ignore the back-scattered waves since in many applications in lossy media, for example, tissue imaging, these waves are too weak and are likely to be masked by noise and uncertainties in the measurements. Also, the measurement system may be nonreciprocal due to the use of amplifiers (or other nonreciprocal components) at the transmitting and/or receiving sides. This makes the measurement of the reflected signals impossible unless separate $\mathrm{Tx}$ and $\mathrm{Rx}$ channels are provided.

In $[19,20]$, both antennas are assumed to be always in the same $\left(x^{\prime}, y^{\prime}\right)$ position during the scan. Here, we consider the general case where antenna 2 may have an offset of $\pm D_{x}$ 
and $\pm D_{y}$ with respect to antenna 1 along the $x$ - and $y$-axes, respectively.

In the following, we study the spatial resolution limits of the two-antenna imaging setup employing an approach previously proposed for bistatic SAR [24]. Note that the resolution limits derived with this method (which assumes propagating waves) provide good estimates in the case of the two-antenna holographic setup where the object is in the far-field region of the antennas. However, in the nearfield imaging it is possible to obtain better resolution due to the availability of evanescent waves. The closer the antennas are to the object, the better the resolution is in a near-field imaging scenario.

With reference to Figure 1 , at each sampling position $\left(x^{\prime}, y^{\prime}, 0\right)$ for antenna 1 -this implies that antenna 2 is at $\left(x^{\prime}+D_{x}, y^{\prime}+D_{y}, \bar{z}\right)$-the total path traveled by the wave through the object is denoted by $R$ and is written as

$$
R=R_{1}+R_{2}
$$

where

$$
\begin{gathered}
R_{1}=\sqrt{\left(x-x^{\prime}\right)^{2}+\left(y-y^{\prime}\right)^{2}+z^{2}} \\
R_{2}=\sqrt{\left[\left(x^{\prime}+D_{x}\right)-x\right]^{2}+\left[\left(y^{\prime}+D_{y}\right)-y\right]^{2}+(\bar{z}-z)^{2}} .
\end{gathered}
$$

The differential change in $R$, denoted by $\Delta R$, due to an incremental change $\left(\Delta \rho_{s} \widehat{\mathbf{s}}\right)$ in the position of the object in any arbitrary direction $\widehat{\mathbf{s}}$, is written as [24]

$$
\Delta R=\left(\widehat{\mathbf{s}}^{T} \cdot \nabla R\right) \Delta \rho_{s},
$$

where $\nabla$ is the gradient operator and the superscript $T$ represents the transpose operator. Here, $\widehat{\mathbf{s}}$ is a unit vector.

On the other hand, the minimum "measureable" $\Delta R$ is related to the velocity of the wave in the medium $c$ and the bandwidth of the imaging system $B$ as [24]

$$
\Delta R_{\min } \approx \frac{c}{B} \text {. }
$$

From (3) and (4), the resolution limit in the direction $\widehat{\mathbf{s}}$, denoted by $\bar{\rho}_{s}$, is estimated as [24]

$$
\bar{\rho}_{s}=\frac{c}{B} \cdot \frac{1}{\left|\widehat{\mathbf{s}}^{T} \nabla R\right|} .
$$

Please note that the resolution in this paper is defined as the half-power (or half-intensity) width of a point-like object.

To estimate the resolution of the imaging system along the $x, y$, and $z$ directions, first the gradient of $R$ in a rectangular coordinate system is written as

$$
\nabla R=\left[\begin{array}{l}
\frac{\partial R}{\partial x} \\
\frac{\partial R}{\partial y} \\
\frac{\partial R}{\partial z}
\end{array}\right]=\left[\begin{array}{c}
\frac{\left(x-x^{\prime}\right)}{R_{1}}-\frac{\left[\left(x^{\prime}+D_{x}\right)-x\right]}{R_{2}} \\
\frac{\left(y-y^{\prime}\right)}{R_{1}}-\frac{\left[\left(y^{\prime}+D_{y}\right)-y\right]}{R_{2}} \\
\frac{z}{R_{1}}-\frac{(\bar{z}-z)}{R_{2}}
\end{array}\right]
$$

Then, from (5) and (6), the cross-range resolution $\bar{\rho}_{u}(u=$ $x$ or $y$ ) and the range resolution $\bar{\rho}_{z}$ are obtained as

$$
\begin{gathered}
\bar{\rho}_{u}=\frac{c}{B} \cdot \frac{1}{\left|\left(\left(u-u^{\prime}\right) / R_{1}\right)-\left(\left[\left(u^{\prime}+D_{u}\right)-u\right] / R_{2}\right)\right|}, \\
u=x, y, \quad u^{\prime}=x^{\prime}, y^{\prime}, \\
\bar{\rho}_{z}=\frac{c}{B} \cdot \frac{1}{\left|\left(z / R_{1}\right)-\left((\bar{z}-z) / R_{2}\right)\right|} .
\end{gathered}
$$

In the previous equations, $\bar{\rho}_{u}$ and $\bar{\rho}_{z}$ are obtained when $\widehat{\mathbf{s}}$ in (5) is substituted with the unit vector in the particular direction, $x, y$, or $z$.

We assume that the object is very small and it is positioned on the $z$-axis that is, the object is at $(0,0, z)$. Then, $(7)$ are simplified as

$$
\begin{gathered}
\bar{\rho}_{u}=\frac{c}{B} \cdot \frac{1}{\left|\left(-u^{\prime} / R_{1}^{\prime}\right)-\left(\left(u^{\prime}+D_{u}\right) / R_{2}^{\prime}\right)\right|}, \\
u^{\prime}=x^{\prime}, y^{\prime}, \quad D_{u}=D_{x}, D_{y}, \\
\bar{\rho}_{z}=\frac{c}{B} \cdot \frac{1}{\left|\left(z / R_{1}^{\prime}\right)-\left((\bar{z}-z) / R_{2}^{\prime}\right)\right|},
\end{gathered}
$$

where

$$
\begin{gathered}
R_{1}^{\prime}=\sqrt{x^{\prime 2}+y^{\prime 2}+z^{2}}, \\
R_{2}^{\prime}=\sqrt{\left(x^{\prime}+D_{x}\right)^{2}+\left(y^{\prime}+D_{y}\right)^{2}+(\bar{z}-z)^{2}} .
\end{gathered}
$$

As observed from the previous equations, the range and cross-range resolution limits depend on the positions of the transceivers and the object.

To derive the resolution limits, we consider a $2 \mathrm{D}$ cut in the $3 \mathrm{D}$ setup in Figure 1 as shown in Figure 2, where the axis $u$ represents either $x$ or $y . D_{u}$ and $u^{\prime}$ denote the offsets of the receiver with respect to the transmitter and the position of the transmitter, respectively.

With reference to Figure 2, we first rewrite the resolution limits in (8) in terms of the angles $\theta_{1}$ and $\theta_{2}$ as

$$
\begin{aligned}
& \bar{\rho}_{u}=\frac{c}{B} \cdot \frac{1}{\left|\sin \theta_{1}+\sin \theta_{2}\right|}, \\
& \bar{\rho}_{z}=\frac{c}{B} \cdot \frac{1}{\left|\cos \theta_{1}-\cos \theta_{2}\right|} .
\end{aligned}
$$

These are the angles of incidence and scattering, respectively, associated with the imaged point-like object.

From (11), it follows that when the transmitter and the receiver are at the same position $\left(x^{\prime}, y^{\prime}\right)$ during the $2 \mathrm{D}$ scan (as in $[19,20]$ ), that is, when $D_{u}=0$, there are two main regions in the inspected volume where the range resolution $\bar{\rho}_{z}$ is poor. These two regions are (1) the middle of the inspected domain, that is, $z \approx \bar{z} / 2$ and (2) the direct path connecting the transceivers when it passes right through the scatterer; that is, $\theta_{1} \approx \theta_{2} \approx 0$. In Figure 2 , the regions of poor range resolution are shaded in gray. 


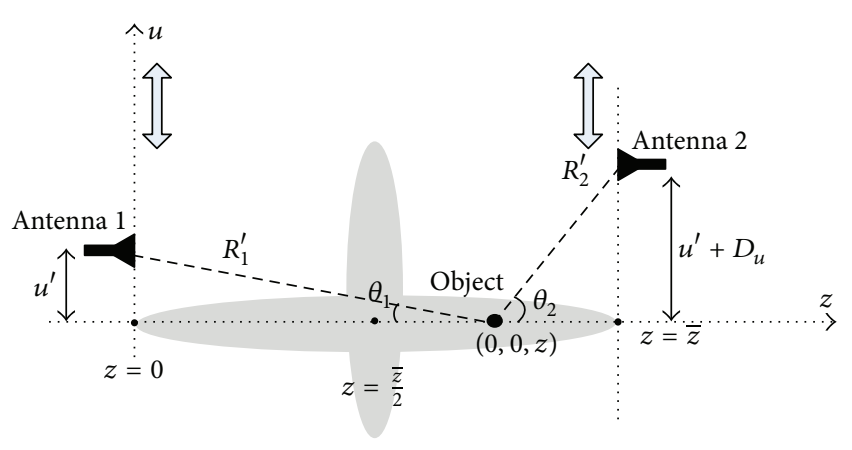

FIGURE 2: Simplifying the 3D setup in Figure 1 to a $2 \mathrm{D}$ setup for deriving resolution limits in terms of $\theta_{1}$ and $\theta_{2}$. When $D_{u}=0$, the range resolution is poor in the shaded region. The grey thick arrows show the directions of the scan.

On the other hand, when the receiver has an offset with respect to the transmitter, that is, when $D_{u} \neq 0$, we obtain finite values for both the cross-range and the range resolution limits everywhere in the inspected volume. In this configuration, as per (10), the best cross-range resolution is obtained when the antennas are at the edges of the apertures; that is, the angles $\theta_{1}$ and $\theta_{2}$ are close to $\pi / 2$ :

$$
\left.\bar{\rho}_{u}^{\min }\right|_{\theta_{1}=\theta_{2}= \pm(\pi / 2)}=\frac{c}{2 B} .
$$

Regarding the cross-range resolution, as per (10), it is best when one antenna is on the $z$-axis $\left(\theta_{1}=0\right)$ while the other antenna is at $u \rightarrow \infty\left(D_{u} \rightarrow \infty, \theta_{2} \rightarrow \pi / 2\right)$ :

$$
\bar{\rho}_{u}=\frac{c}{B} \text {. }
$$

Under these conditions, the range resolution is

$$
\left.\bar{\rho}_{z}^{\min }\right|_{\substack{\theta_{1}=0, \theta_{2}= \pm \pi / 2 \\ \theta_{1}= \pm \pi / 2, \theta_{2}=0}}=\frac{c}{B} .
$$

It is worth noting that in reflection holography where $\theta_{2}=$ $\pi-\theta_{1}$, the cross-range and range resolution limits are

$$
\begin{aligned}
& \bar{\rho}_{u}=\frac{c}{B} \cdot \frac{1}{\left|2 \sin \theta_{1}\right|}, \\
& \bar{\rho}_{z}=\frac{c}{B} \cdot \frac{1}{\left|2 \cos \theta_{1}\right|} .
\end{aligned}
$$

Assuming $B \approx 2 f_{c}$ with $f_{c}$ being the center frequency of the band, the cross-range resolution can be written as follows:

$$
\bar{\rho}_{u}=\frac{\lambda_{c}}{4 \sin \theta_{1}}
$$

where $\lambda_{c}$ is the wavelength at $f_{c}$. Thus, in this case, the crossrange resolution improves if the size of the aperture is large $\left(\theta_{1} \rightarrow \pi / 2\right)$. On the other hand, the best range resolution limit is obtained when $\theta_{1} \rightarrow 0$ as

$$
\bar{\rho}_{z}^{\min }=\frac{c}{2 B} .
$$

These results are consistent with the expressions for the resolution limits of reflection holography in [11].

\section{Multiple Receiver Setup}

In the previous section, we showed that having a nonzero offset distance for the receiver along the $x$ - or $y$-axis $\left(D_{u} \neq 0\right.$, $u=x, y$ ) leads to improving the range and cross-range resolutions in a scenario where forward-scattered signals are acquired. Thus, here we propose a multiple receiver setup in a star distribution to achieve satisfactory range and crossrange resolutions in 3D microwave imaging with planar raster scanning.

Figure 3 illustrates the setup where one transmitter illuminates the object while five receivers measure the forwardscattered waves. Antenna 2 is aligned with the transmitter (antenna 1) while the other receivers (antennas 3 to 6) have offset distances of $\pm D_{x}$ and $\pm D_{y}$ along the $\pm x$ and $\pm y$ directions, respectively. The transmitter and the five receivers move together during the $2 \mathrm{D}$ scan on the two planar apertures. From the results in Section 2, it follows that larger offset distances lead to improved resolution. However, at large offset distances the scattered wave travels along longer paths from the object to the receiver. This weakens the signal due to two factors: spatial spread and attenuation if the medium is lossy. Besides, signal strength can also weaken due to the antenna pattern. These factors impose upper limits on the offset distances. In addition, increasing the offset distances increases the size of aperture 2 . If the size of aperture 1 is $2 L_{x} \times$ $2 L_{y}$, then the size of aperture 2 is $2\left(L_{x}+D_{x}\right) \times 2\left(L_{y}+D_{y}\right)$.

\section{3D Holographic Image Reconstruction}

In this section, we extend our previously proposed 3D holographic microwave imaging algorithm [19] to process the data obtained with the multiple receiver configuration proposed here.

With reference to Figure 3, the transmitting antenna (antenna 1) and the receiving antenna array (antennas 2 to 6) perform a $2 \mathrm{D}$ scan at the planes $z=0$ and $z=\bar{z}$, respectively. For simplicity, we assume that the antennas are $x$-polarized (e.g., dipoles oriented along the $x$-axis). Thus, the field can be reasonably approximated by a $\mathrm{TM}_{x}$ polarization and the theory is scalar in nature (the algorithm for a full-vector $3 \mathrm{D}$ holography is available in [20]). The approach here is directly applicable to acquisition of co- and cross-polarized data. In the scalar case, Green's function $G$ can be viewed as the $G_{x}^{x}$ element of the full dyadic while the $E$-field is represented by its $x$-component only. From now on we omit the subscript $x$ for brevity.

Assume that at any measurement frequency $\omega_{l}(l=$ $\left.1,2, \ldots, N_{\omega}\right)$ we know the incident field $E_{\text {inc }}\left(0,0,0 ; x, y, z ; \omega_{l}\right)$ at any point $(x, y, z)$ in the inspected volume due to antenna 1 when it is at $(0,0,0)$. In addition, Green's function for antenna $2 G_{2}\left(x, y, z ; 0,0, \bar{z} ; \omega_{l}\right)$ is known for an $x$-polarized scattering point source at $(x, y, z)$ and the $x$-polarized response at $(0,0, \bar{z})$. This information can be obtained via simulations. For brevity, we introduce the notations

$$
\begin{gathered}
E_{\mathrm{inc}}\left(x, y, z, \omega_{l}\right) \equiv E_{\mathrm{inc}}\left(0,0,0 ; x, y, z ; \omega_{l}\right), \\
G_{2}\left(x, y, z, \omega_{l}\right) \equiv G_{2}\left(x, y, z ; 0,0, \bar{z} ; \omega_{l}\right)
\end{gathered}
$$




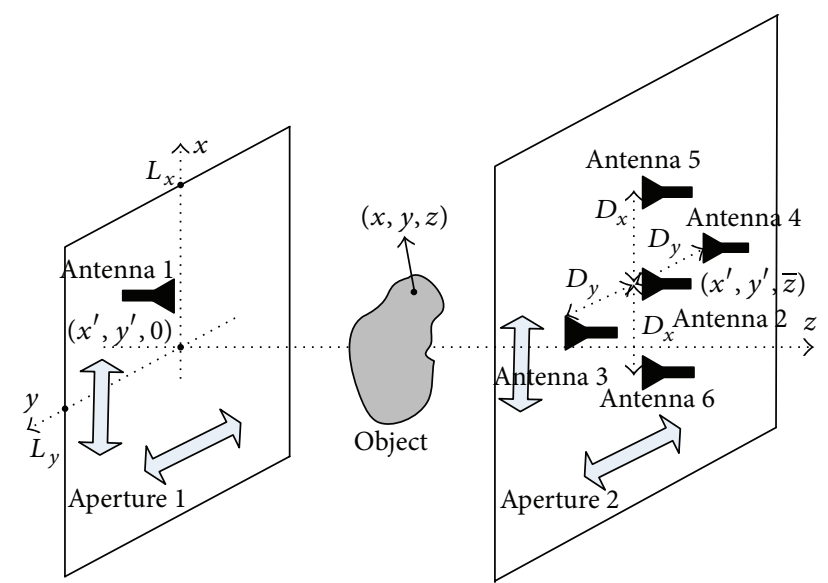

FIGURE 3: Proposed 3D microwave holography setup using forwardscattered waves only. Antenna 1 illuminates the object while antennas 2 to 6 receive the scattered waves. The grey thick arrows show the directions of the scan.

In [20], we show that while $E_{\text {inc }}$ can be obtained from simulations, Green's function can be obtained from $E_{\text {inc }}$ using the reciprocity principle. Thus, assuming that antennas 1 to 6 are identical, only one simulation suffices to obtain $E_{\text {inc }}\left(x, y, z, \omega_{l}\right)$, from which Green's functions for antennas 2 to 6 are obtained as

$$
\begin{gathered}
G_{2}\left(x, y, z, \omega_{l}\right)=E_{\mathrm{inc}}\left(x, y, \bar{z}-z, \omega_{l}\right), \\
G_{3}\left(x, y, z, \omega_{l}\right)=E_{\mathrm{inc}}\left(x, y-D_{y}, \bar{z}-z, \omega_{l}\right), \\
G_{4}\left(x, y, z, \omega_{l}\right)=E_{\mathrm{inc}}\left(x, y+D_{y}, \bar{z}-z, \omega_{l}\right), \\
G_{5}\left(x, y, z, \omega_{l}\right)=E_{\mathrm{inc}}\left(x-D_{x}, y, \bar{z}-z, \omega_{l}\right), \\
G_{6}\left(x, y, z, \omega_{l}\right)=E_{\mathrm{inc}}\left(x+D_{x}, y, \bar{z}-z, \omega_{l}\right) .
\end{gathered}
$$

Let $E_{k}^{s c}\left(x^{\prime}, y^{\prime}, \bar{z}, \omega_{l}\right)$ be the scattered $E$-field received by the $k$ th antenna $(k=2, \ldots, 6)$ when the transmitting antenna is at $\left(x^{\prime}, y^{\prime}, 0\right)$. Following the approach presented in [19], $E_{k}^{s c}\left(x^{\prime}, y^{\prime}, \bar{z}, \omega_{l}\right)$ is written as

$$
\begin{array}{r}
E_{k}^{\mathrm{sc}}\left(x^{\prime}, y^{\prime}, \bar{z}, \omega_{l}\right) \\
=\int_{z} \int_{y} \int_{x} f(x, y, z) \\
\cdot a_{k}\left(x^{\prime}-x, y^{\prime}-y, z, \omega_{l}\right) d x d y d z, \\
\text { for } k=2, \ldots, 6,
\end{array}
$$

where $f(x, y, z)=k_{s}^{2}(x, y, z)-k_{b}^{2}$ is the contrast function, $k_{s}$ and $k_{b}$ are the wavenumbers of the object and the background mediums, respectively, and

$$
a_{k}\left(x, y, z, \omega_{l}\right)=E_{\mathrm{inc}}\left(-x,-y, z, \omega_{l}\right) G_{k}\left(-x,-y, z, \omega_{l}\right) .
$$

Notice that in (20), the integration over $x$ and $y$ can be interpreted as a $2 \mathrm{D}$ convolution integral. Thus, the $2 \mathrm{D}$ FT of $E_{k}^{\text {sc }}$ is written as

$$
\begin{array}{r}
\widetilde{E}_{k}^{\text {sc }}\left(k_{x}, k_{y}, \bar{z}, \omega_{l}\right)=\int_{z} \widetilde{F}\left(k_{x}, k_{y}, z\right) \widetilde{A}_{k}\left(k_{x}, k_{y}, z, \omega_{l}\right) d z \\
\text { for } k=2, \ldots, 6,
\end{array}
$$

where $\widetilde{F}$ and $\widetilde{A}_{k}$ are the $2 \mathrm{D}$ FTs of $f(x, y, z)$ and $a_{k}\left(x, y, z, \omega_{l}\right)$, respectively. By approximating the integral in (22) by a discrete sum and employing the data collected at all frequencies and at all receivers, we construct a system of equations at each $\left(k_{x}, k_{y}\right)$ as

$$
\underline{\widetilde{\mathbf{E}}}=\underline{\underline{\mathbf{A}}} \underline{\widetilde{\mathbf{F}}},
$$

where

$$
\underline{\widetilde{\mathbf{E}}}=\left[\begin{array}{c}
{\left[\underline{\widetilde{E}}_{2}^{s c}\right]} \\
\vdots \\
{\left[\widetilde{\underline{E}}_{6}^{s c}\right]}
\end{array}\right], \quad \underline{\underline{\mathbf{A}}}=\left[\begin{array}{c}
{\left[\widetilde{\widetilde{A}}_{2}\right]} \\
\vdots \\
{[\widetilde{\widetilde{A}}} \\
\left.\underline{\underline{G}}_{6}\right]
\end{array}\right], \quad \underline{\widetilde{\mathbf{F}}}=\left[\begin{array}{c}
\widetilde{F}\left(z_{1}\right) \\
\vdots \\
\widetilde{F}\left(z_{N_{z}}\right)
\end{array}\right],
$$

and, for each $k=2, \ldots, 6$,

$$
\begin{gathered}
{\left[\underline{\widetilde{E}}_{k}^{s c}\right]=\left[\begin{array}{c}
\widetilde{E}_{k}^{s c}\left(\bar{z}, \omega_{1}\right) \\
\vdots \\
\widetilde{E}_{k}^{s c}\left(\bar{z}, \omega_{N_{\omega}}\right)
\end{array}\right],} \\
{\left[\underline{\underline{A}}_{k}\right]=\left[\begin{array}{ccc}
\widetilde{A}_{k}\left(z_{1}, \omega_{1}\right) \Delta z & \cdots & \widetilde{A}_{k}\left(z_{N_{z}}, \omega_{1}\right) \Delta z \\
\vdots & \vdots & \vdots \\
\widetilde{A}_{k}\left(z_{1}, \omega_{N_{\omega}}\right) \Delta z & \cdots & \widetilde{A}_{k}\left(z_{N_{z}}, \omega_{N_{\omega}}\right) \Delta z
\end{array}\right] .}
\end{gathered}
$$

Here, $\Delta z$ is the discretization step along the $z$-axis. In (24) and (25), the arguments $k_{x}$ and $k_{y}$ have been omitted for brevity.

The system in (23) is solved at each $\left(k_{x}, k_{y}\right)$ pair for $\widetilde{F}\left(z_{n}\right), n=1, \ldots, N_{z}$, in a least-square sense. To reduce the ill-conditioning of the least-square solution we employ the Tikhonov regularization [25]. Thus, the solution is obtained from

$$
\underline{\widetilde{\mathbf{F}}}=\left(\underline{\underline{\mathbf{A}}}^{*} \underline{\underline{\mathbf{A}}}+\underline{\Gamma}^{*} \underline{\underline{\Gamma}}\right)^{-1} \underline{\underline{\mathbf{A}}}^{*} \underline{\widetilde{\mathbf{E}}},
$$

where $(\cdot)^{*}$ is the complex conjugate operator, $\underline{\underline{\Gamma}}=\alpha \mathbf{I}$ is the Tikhonov matrix, $\alpha$ is the Tikhonov regularization parameter, and $\mathbf{I}$ is the identity matrix. The optimal value of the regularization parameter is usually determined by an ad hoc method in practical problems. In this study, we use the plot of the Lagrange function to determine $\alpha$. The MATLAB code for this method is available in MATLAB central [26].

Once the systems of equations for all $\left(k_{x}, k_{y}\right)$ are solved, the inverse 2D FT is applied to $\widetilde{F}\left(k_{x}, k_{y}, z_{n}\right), n=1,2, \ldots, N_{z}$, to reconstruct a 2D slice of the function $f\left(x, y, z_{n}\right)$ at each $z=z_{n}$ plane. Then, the normalized modulus of $f\left(x, y, z_{n}\right)$, 
$\left|f\left(x, y, z_{n}\right)\right| / M$, where $M$ is the maximum of $\left|f\left(x, y, z_{n}\right)\right|$ for all $z_{n}$, is plotted versus the spatial coordinates $x$ and $y$ to obtain 2D images of the object at all $N_{z}$ planes. By putting together all 2D slice images, a $3 \mathrm{D}$ image of the object is obtained.

\section{Image Reconstruction Results}

The performance of the proposed multiple receiver planarscan imaging method is studied through various simulation examples using FEKO [27] and an experimental example. First, we present an example demonstrating the improvement achieved in the multiple receiver configuration compared to the single receiver configuration where $D_{x}=D_{y}=0$. Then, we study the resolution limits of the multiple receiver setup.

As a quick note, the choice of frequency range in microwave imaging of lossy objects is primarily driven by the trade-off between resolution, which improves with increasing frequency and penetration, which usually improves with decreasing frequency. Thus, it has to be chosen properly and in accordance with the properties of the inspected dielectric medium.

5.1. Two-Antenna System versus Six-Antenna System. In the first example, six $\lambda / 2$ (at $6.5 \mathrm{GHz}$ ) $x$-polarized dipole antennas are employed to scan two cuboids as shown in Figure 4. The objects have a side of $2 \mathrm{~mm}$ and center-to-center distance of $8 \mathrm{~mm}$. They are at the range position of $z=30 \mathrm{~mm}$. The frequency-independent properties of the background medium are $\varepsilon_{r}^{\mathrm{b}}=16$ and $\sigma_{\mathrm{b}}=0.5 \mathrm{~S} / \mathrm{m}$ while those of the two objects are $\varepsilon_{r}^{o}=32$ and $\sigma_{o}=1 \mathrm{~S} / \mathrm{m}$. Antenna 2 is aligned with Antenna 1 (transmitter). Antennas 3 to 6 have offsets of $\pm D_{x}$ and $\pm D_{y}$ along the $\pm x$ and $\pm y$ directions, respectively, as illustrated in Figure 4. Antenna 1 scans an area of $120 \mathrm{~mm} \times$ $120 \mathrm{~mm}$. Thus, with reference to Figure 3, aperture 1 has a size of $120 \mathrm{~mm} \times 120 \mathrm{~mm}$ while aperture 2 has a size of $(120 \mathrm{~mm}+$ $\left.2 D_{x}\right) \times\left(120 \mathrm{~mm}+2 D_{y}\right)$. The number of samples along both $x$ and $y$ directions is 61 .

The transmission $S$-parameters $S_{k 1}(k=2$ to 6$)$ are acquired over the frequency range from $3 \mathrm{GHz}$ to $10 \mathrm{GHz}$. The sampling rate in frequency is determined based on the Nyquist criterion as suggested in $[11,19]$. The maximum phase shift that results from a change in the wavenumber $\Delta k$ is $\Delta k R_{\max }$, where $R_{\max }$ is the maximum distance traveled by the wave from the transmitter to the receiver through the object. Requiring that this phase shift be less than $\pi$ rad yields

$$
\Delta f<\frac{c}{2 R_{\max }} .
$$

Here, a sampling step of $\Delta f=0.25 \mathrm{GHz}$ is chosen which is approximately $c / 2 R_{\max }$ with $R_{\max }=150 \mathrm{~mm}$. In general, based on our studies, decreasing $\Delta f$ further improves the robustness to noise to some extent.

When calibrated (as described in [19]), the $S_{k 1}$ (for $k=$ $2, \ldots, 6)$ parameters provide good approximations of the $x$ component of the forward-scattered waves at the center of the antennas.

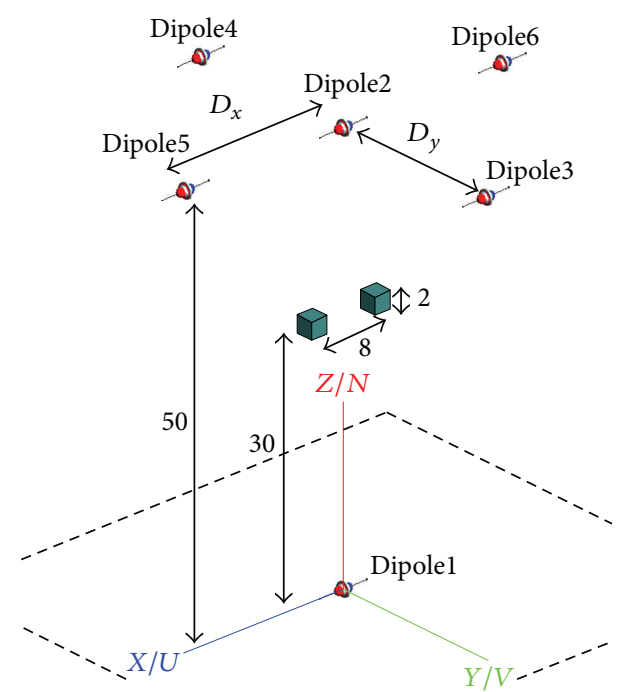

FIgURE 4: Simulation example in FEKO. All dimensions are in $\mathrm{mm}$. The background has properties of $\varepsilon_{r}^{\mathrm{b}}=16$ and $\sigma_{\mathrm{b}}=0.5 \mathrm{~S} / \mathrm{m}$. Imaging is performed for two objects along the $x$ axis with properties $\varepsilon_{r}^{o}=32$ and $\sigma_{\mathrm{o}}=1 \mathrm{~S} / \mathrm{m}$.

We first show that using the forward-scattered waves acquired in a single receiver configuration when $D_{x}=$ $D_{y}=0$ is not sufficient to implement $3 \mathrm{D}$ holography as we discussed in Section 2. Figure 5(a) shows the reconstructed images when only $S_{21}$ is processed using the $3 \mathrm{D}$ holography technique presented in [19]. The quality of the reconstructed images is not satisfactory. Not only the two objects are not resolved well at $z=30 \mathrm{~mm}$ (their true range location) but also the images at other range locations show the presence of strong artifacts.

Then, we process the forward-scattered waves sampled by the five offset receivers and apply the reconstruction technique proposed here. Figure 5(b) shows the images obtained when all transmission $S$-parameters $\left(S_{k 1}, k=2, \ldots, 6\right)$ are employed and when $D_{x}=D_{y}=10 \mathrm{~mm}$. As the figures show, the two objects are more discernible in the image at $z=30 \mathrm{~mm}$ and the artifacts in the images at other range locations are much weaker compared to those in Figure 5(a). Figure 5(c) shows the images obtained when $D_{x}=D_{y}=$ $20 \mathrm{~mm}$. It is obvious that increasing the offset distances $D_{x}$ and $D_{y}$ improves the quality of the images as expected from the discussion in Section 2.

5.2. Simulation Examples for the Six-Antenna System. We conduct FEKO simulations for the setups illustrated in Figures 6(a)-6(d).

In the first example (Figure 6(a)), a single small object is present, which allows the study of the point-spread function (PSF) of the method. The distance between the two apertures is $70 \mathrm{~mm}$ and one cuboid object is placed midway between them. The size and properties of the object and the properties of the background medium are the same as in the previous example. The offset distances are $D_{x}=D_{y}=20 \mathrm{~mm}$. The data acquisition process and sampling in space and frequency is 

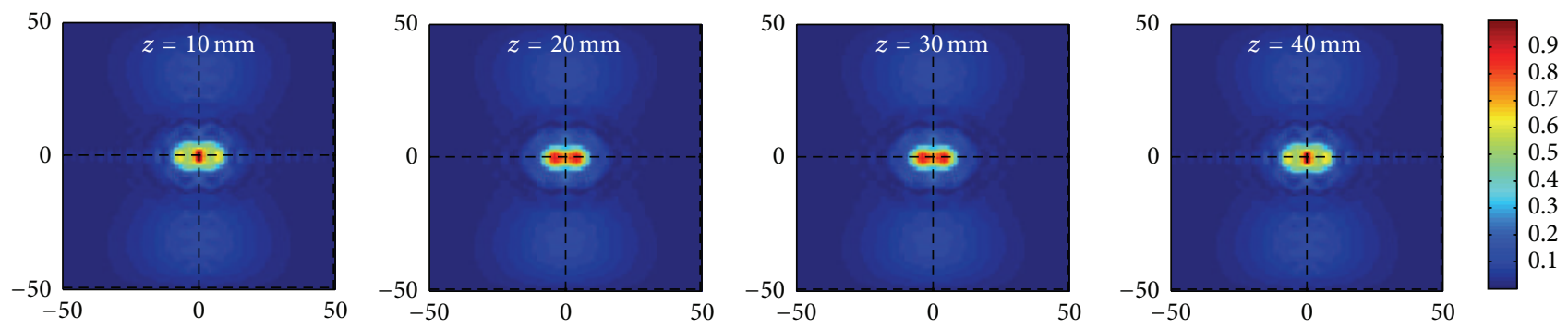

(a)
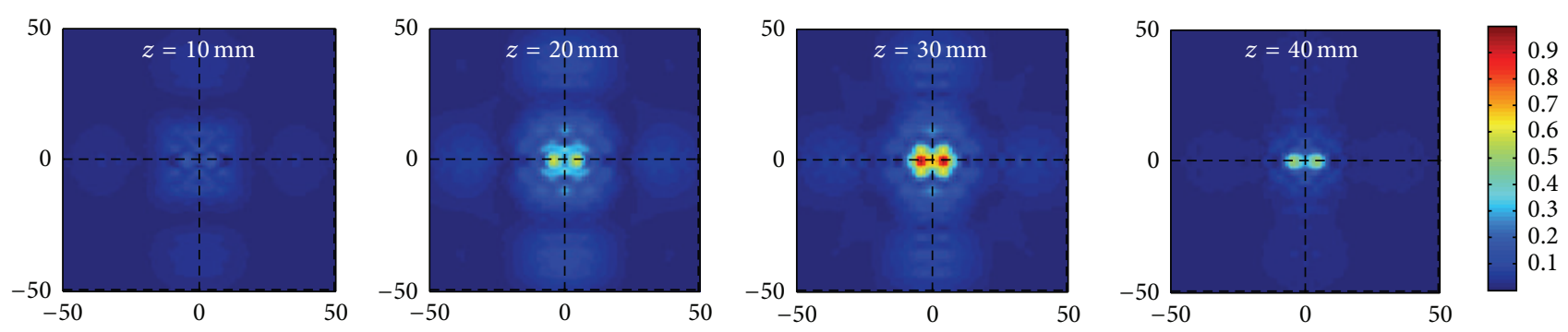

(b)
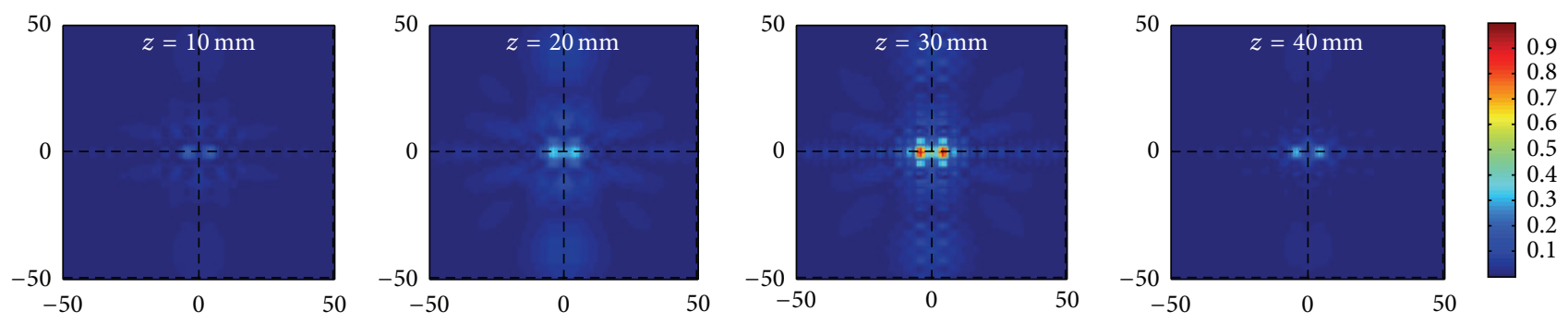

(c)

Figure 5: Reconstructed images for the objects shown in Figure 4 when applying 3D microwave holography to (a) only $S_{21}$, (b) all five transmission $S$-parameters when $D_{x}=D_{y}=10 \mathrm{~mm}$, and (c) all five transmission $S$-parameters when $D_{x}=D_{y}=20 \mathrm{~mm}$. The horizontal and vertical axes show the positions along the $x$ and $y$ directions in terms of $\mathrm{mm}$.

the same as in the previous example. To keep the simulations of the $2 \mathrm{D}$ scan affordable in terms of computational time, we have used a relatively coarse mesh size of $\lambda / 10$, where $\lambda$ is the wavelength at $6.5 \mathrm{GHz}$ in the background medium. To estimate the signal-to-noise ratio (SNR) of the simulations, we also perform a single simulation with a mesh size of $\lambda / 30$ and evaluate the change in the magnitude of the transmission $S$-parameter between Antenna 1 and Antenna 2, that is, $S_{21}$. If we denote $S_{21}$ obtained with the coarse and fine meshes as $S_{21}^{\mathrm{c}}$ and $S_{21}^{\mathrm{f}}$, respectively, then we evaluate the numerical SNR at each frequency $\omega$ as follows:

$$
\operatorname{SNR}(\omega)=20 \log \left[\frac{\left|S_{21}^{\mathrm{f}}(\omega)\right|}{\left|S_{21}^{\mathrm{f}}(\omega)-S_{21}^{\mathrm{c}}(\omega)\right|}\right]
$$

Figure 7 shows the variation of SNR with frequency. The minimum SNR is as low as $-5 \mathrm{~dB}$ at the lower frequencies. The maximum SNR is still low and around $12 \mathrm{~dB}$ in the middle of the frequency band. For image reconstruction, we intentionally use the set of data acquired with the coarse mesh to show the performance of the algorithm in the presence of high levels of noise and uncertainties. We note that the variation of the SNR versus frequency is similar in all other simulation examples presented here.

Figure 8(a) shows the images reconstructed with $\Delta z$ $=5 \mathrm{~mm}$ and with the Tikhonov regularization parameter $\alpha$ being $2.5 \times 10^{-5}$ (the same value is used in the other simulation examples). For brevity we only show images every $10 \mathrm{~mm}$. It is observed that the object is recovered well at $z=35 \mathrm{~mm}$ and the artifacts at other range positions are weak. Figures 8(b) and 8(c) show the $1 \mathrm{D}$ images of this object along the $z$-axis and also along the $x$-and $y$-axes in the $z=35 \mathrm{~mm}$ plane. From the half-power width of these functions, we estimate the range and cross-range resolutions to be $\bar{\rho}_{z}=12 \mathrm{~mm}$ and $\bar{\rho}_{u}=4 \mathrm{~mm}$. These are close to the values computed from (12) and (14), that is, $\bar{\rho}_{z}^{\text {min }}=10.8 \mathrm{~mm}$ and $\bar{\rho}_{u}^{\min }=5.4 \mathrm{~mm}$. One may argue that the offsets are not very large in this example $\left(D_{x}=D_{y}=20 \mathrm{~mm}\right)$ and the resolution limits should not be close to the resolution limits computed using (12) and (14). Similar conclusions are drawn here from another example, where $\bar{z}=50 \mathrm{~mm}$. We believe that the slight improvement in the resolution is due to the combination of data obtained from various look angles (we have five receivers and for each receiver the data 


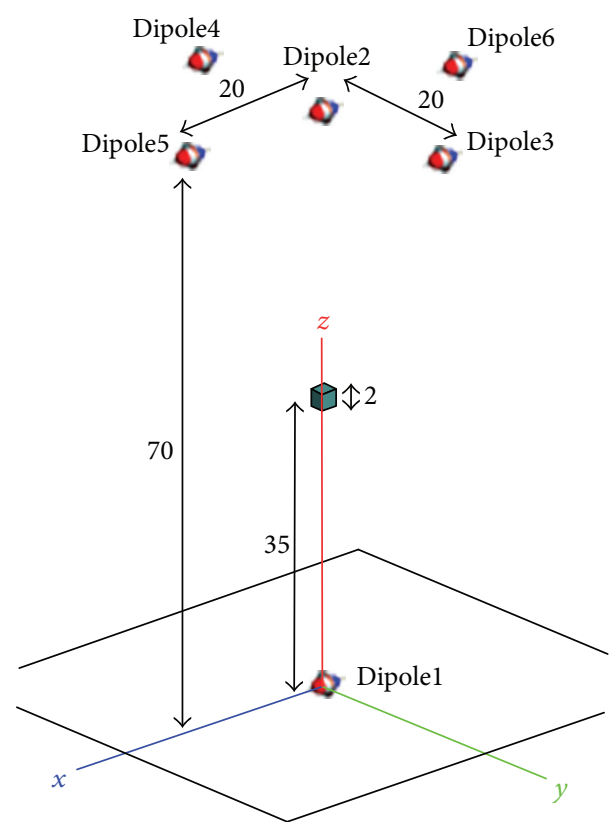

(a)

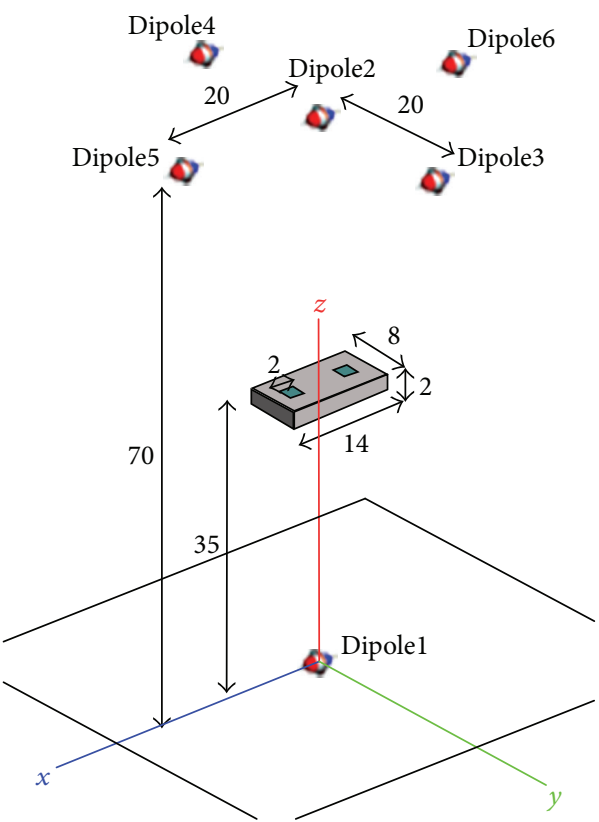

(c)

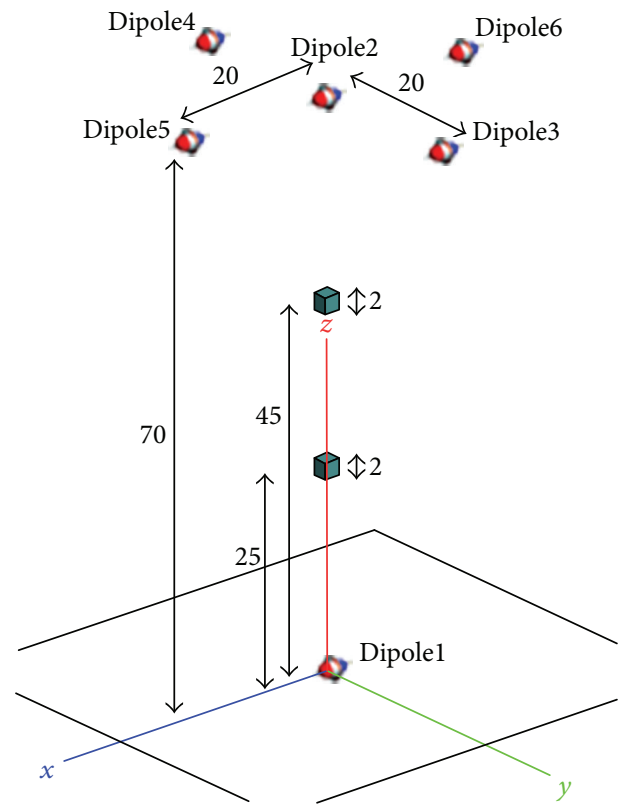

(b)

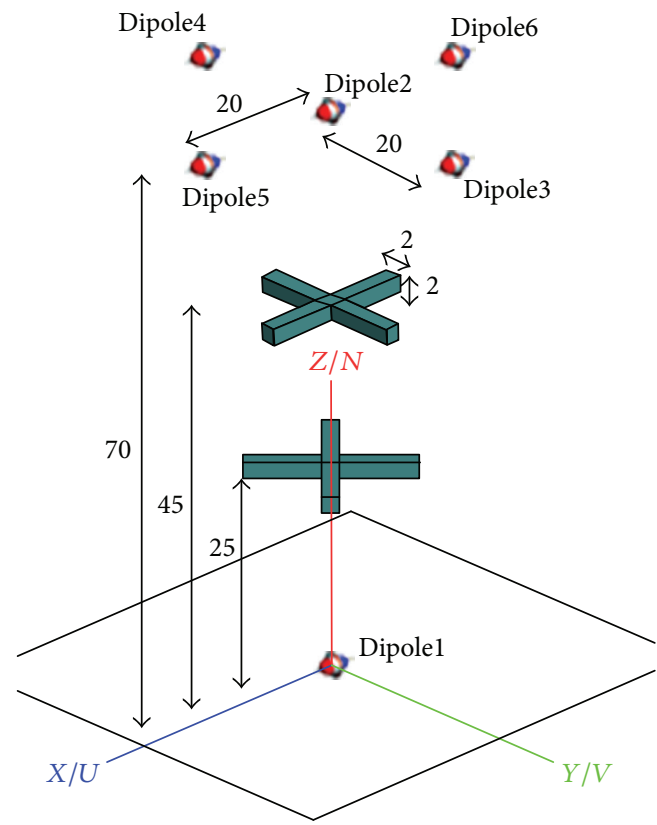

(d)

FIGURE 6: Simulation examples in FEKO. The background has properties of $\varepsilon_{r}^{\mathrm{b}}=16$ and $\sigma_{\mathrm{b}}=0.5 \mathrm{~S} / \mathrm{m}$. Imaging is performed for (a) a single object with properties $\varepsilon_{r}^{o}=32$ and $\sigma_{\mathrm{o}}=1 \mathrm{~S} / \mathrm{m}$, (b) two objects with properties $\varepsilon_{r}^{\mathrm{o}}=32$ and $\sigma_{\mathrm{o}}=1 \mathrm{~S} / \mathrm{m}$ along the $z$-axis, (c) two objects with properties $\varepsilon_{r}^{0}=48$ and $\sigma_{\mathrm{o}}=2 \mathrm{~S} / \mathrm{m}$ along the $x$-axis embedded in a lower contrast medium with properties $\varepsilon_{r}^{\mathrm{m}}=32$ and $\sigma_{\mathrm{m}}=1 \mathrm{~S} / \mathrm{m}$, and $(\mathrm{d})$ two X-shape objects with properties $\varepsilon_{r}^{0}=32$ and $\sigma_{o}=1 \mathrm{~S} / \mathrm{m}$. All dimensions are in $\mathrm{mm}$.

is collected over the aperture). It is wellknown that in the diffraction tomographic imaging techniques based on many viewing angles, the resolution is improved compared to the diffraction-limited resolution [28]).

Also, it is observed that the resolution is improved compared to the diffraction-limited resolution in free space. This is due to the wavelength contraction resulting from the high relative dielectric constant of the background medium.
For example, the diffraction-limited cross-range resolution in free space is approximately $24 \mathrm{~mm}$ while the obtained resolution is $4 \mathrm{~mm}$.

Figure 6(b) shows the setup for another simulation example in which two small objects are along the $z$-axis positioned at $z=25 \mathrm{~mm}$ and $z=45 \mathrm{~mm}$. The properties of the background medium and objects and the dimensions and sampling rates are the same as in the previous examples. 


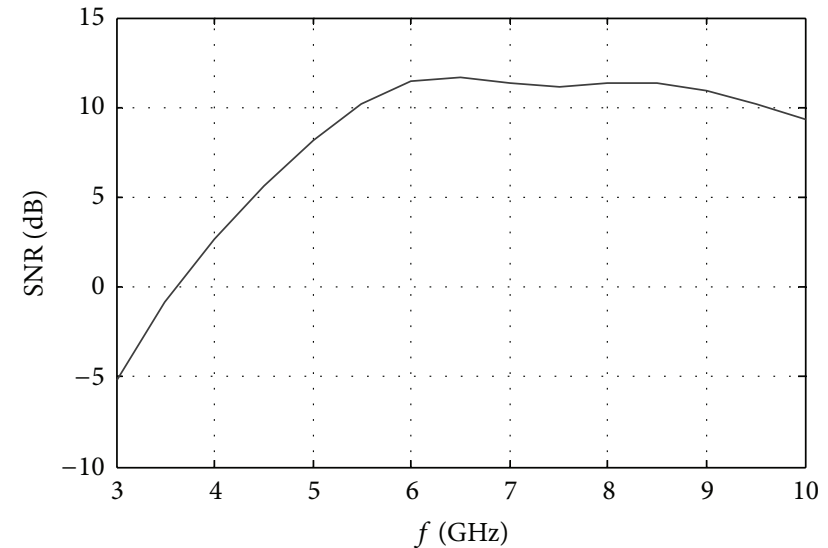

FIGURE 7: Variation of the numerical SNR versus frequency in the simulations.

The reconstruction in this case is more challenging due to the multiple scattering between the two objects and because they are at the same $x$ and $y$ coordinates. Figure 9(a) shows the reconstructed images for this example. It is observed that the two objects are reconstructed at their true locations with weak artifacts at other planes. Figure 9(b) shows the magnitude of the image values along the $z$-axis. It is again observed that the two objects are detected well with the dip between the two peaks dropping below 0.7 level. Figure 9(c) also shows the magnitude of the image values along the $x$-and $y$-axes on the $z=25 \mathrm{~mm}$ plane (similar variation is observed at $z=45 \mathrm{~mm}$ plane).

Figure 6(c) shows the next simulation example in which two cuboids with size of $2 \mathrm{~mm} \times 2 \mathrm{~mm} \times 2 \mathrm{~mm}$ are along the $x$-axis positioned at $x=-4 \mathrm{~mm}$ and $x=4 \mathrm{~mm}$. The properties of the background medium as well as the dimensions, and the spatial and frequency sampling rates are the same as in the previous examples. However, the objects have properties of $\varepsilon_{r}^{o}=48$ and $\sigma_{o}=2 \mathrm{~S} / \mathrm{m}$. They are embedded in a lower contrast medium which is shaped like a box of size $14 \mathrm{~mm} \times 8 \mathrm{~mm} \times 2 \mathrm{~mm}$ and has properties of $\varepsilon_{r}^{\mathrm{m}}=32$ and $\sigma_{\mathrm{m}}=1 \mathrm{~S} / \mathrm{m}$. Figure 10(a) shows the reconstructed images in this example. It is observed that the two objects are reconstructed at their true locations with a shadow around them which represents the lower contrast medium. The images at other range locations show some weak artifacts. Figure 10(b) shows the magnitude of the image values along the $z$-axis. It is observed that the objects are detected well at their true location of $z=35 \mathrm{~mm}$. Figure 10(c) also shows the magnitude of the image values along the $x$ and $y$-axes on the $z=35 \mathrm{~mm}$ plane. It is observed again that the two objects are detected well at their true locations.

Figure 6(d) shows the last simulation example in which two X-shape objects are at the range positions of $25 \mathrm{~mm}$ and $45 \mathrm{~mm}$. The properties of the background medium as well as the dimensions and the spatial and frequency sampling rates are the same as in the previous examples. The objects have electrical properties of $\varepsilon_{r}^{o}=32$ and $\sigma_{\mathrm{o}}=1 \mathrm{~S} / \mathrm{m}$. Figure 11 shows the reconstructed images in this example. It is observed that the two objects are reconstructed at their true range locations with a weaker artifact appearing at $z=35 \mathrm{~mm}$. The images at other range locations show some weak artifacts.

5.3. Experimental Results. We also demonstrate the capabilities of the proposed multiple receiver approach through an experiment in free space. We emphasize that confirming the capability of 3D imaging in free space would also imply the capability of 3D imaging in lossy mediums as long as the SNR is sufficient for reliable reconstruction using transmission coefficients. Besides, 3D imaging in free space itself is important when the setup is nonreciprocal and reflection coefficients cannot be acquired (e.g., due to the use of power amplifiers at the transmitter side or low-noise amplifiers at the receiver side).

Here, six X-band open-ended waveguides are employed, one as a transmitter and five as receivers (as shown in Figure 12). The waveguides have a flange of size $40 \mathrm{~mm} \times$ $40 \mathrm{~mm}$. Thus, for the receivers we have $D_{x}=D_{y}=40 \mathrm{~mm}$. The distance between the two apertures is $\bar{z}=110 \mathrm{~mm}$. The object is a very thin copper sheet with size of $40 \mathrm{~mm} \times 20 \mathrm{~mm}$ centered at $(0,0,58) \mathrm{mm}$ with its edges along the $y$ - and $x$ axes. The antennas are stationary while the object is scanned over a $2 \mathrm{D}$ region of size $160 \mathrm{~mm} \times 200 \mathrm{~mm}$ with steps of $5 \mathrm{~mm}$ along $x$ and $y$. The motion of the object is controlled by a computer through two stepper motors. At each scanning step, the five transmission $S$-parameters $\left(S_{k 1}, k=2, \ldots, 6\right)$ are recorded by an Advantest R3770 vector network analyzer via an RF switch. The measurements are performed in anechoic chamber to minimize the electromagnetic interference.

The $2 \mathrm{D}$ scan is performed two times, once with the object and a second time without the object, in order to calibrate the acquired transmission $S$-parameters as described in [19].

The structure of a commercially available X-band waveguide, which also contains a flange, is modeled in FEKO to obtain the incident field/Green's functions for the transmitter/receivers. Also, to better align the strengths of the simulated fields and the ones produced by the waveguide in practice, we calibrate the simulated incident field as

$$
\begin{array}{r}
E_{\mathrm{inc}, k}^{\mathrm{cal}}(\mathbf{r}, \omega)=\sqrt{\frac{1-\left|S_{k k}^{\text {meas }}(\omega)\right|^{2}}{1-\left|S_{k k}^{\operatorname{sim}}(\omega)\right|^{2}}} E_{\mathrm{inc}, k}^{\mathrm{sim}}(\mathbf{r}, \omega), \\
\text { for } k=2, \ldots, 6,
\end{array}
$$

where $E_{\text {inc }, k}^{\mathrm{cal}}(\mathbf{r}, \omega)$ is the calibrated incident field, $E_{\mathrm{inc}, k}^{\mathrm{sim}}(\mathbf{r}, \omega)$ is the simulated incident field, $\mathbf{r}$ is the position at which the field is evaluated, $S_{k k}^{\text {meas }}(\omega)$ is the measured reflection $S$-parameter, and $S_{k k}^{\text {sim }}(\omega)$ is the simulated reflection $S$-parameter. In effect, (29) aligns the amplitude of the radiated field in the simulations with that in the measurements.

We perform the measurement over a wide band from $3 \mathrm{GHz}$ to $20 \mathrm{GHz}$. Although the antennas are not well matched in the whole band, the signature of the object can be observed and processed from the data collected over the entire band. Figure 13 shows the images obtained with the proposed 3D imaging algorithm. Again, reconstruction is performed with $\Delta z=5 \mathrm{~mm}$ but for brevity we only 

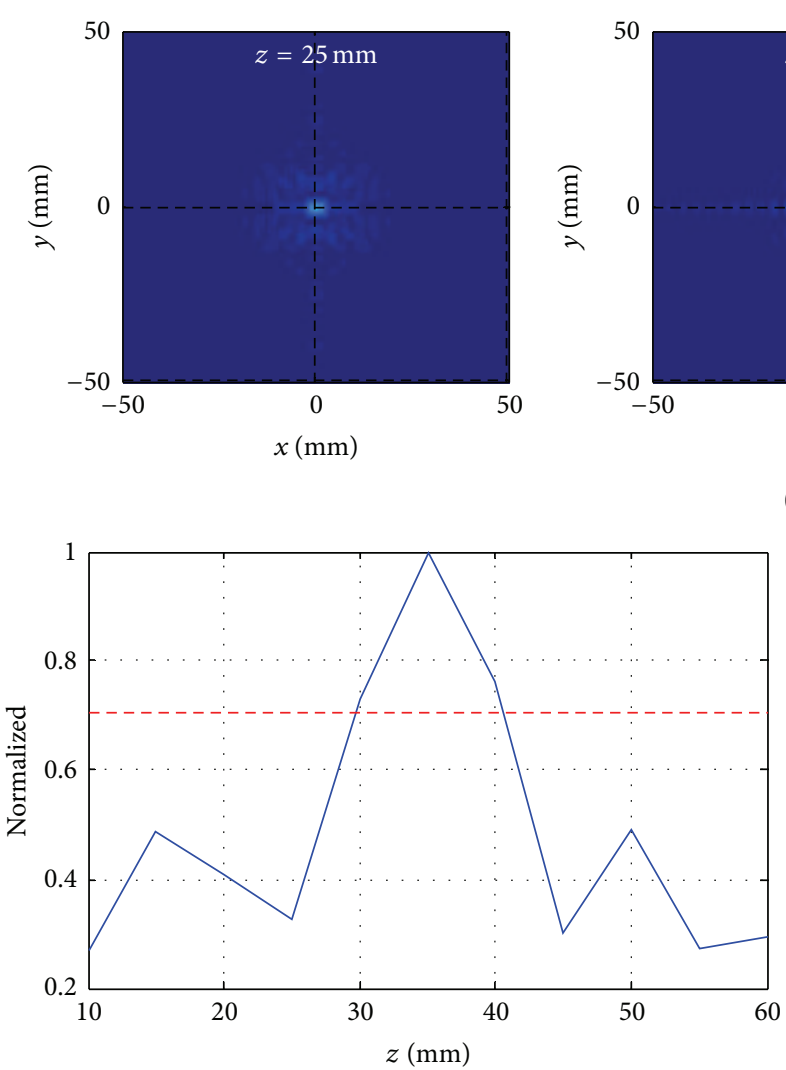

(b)
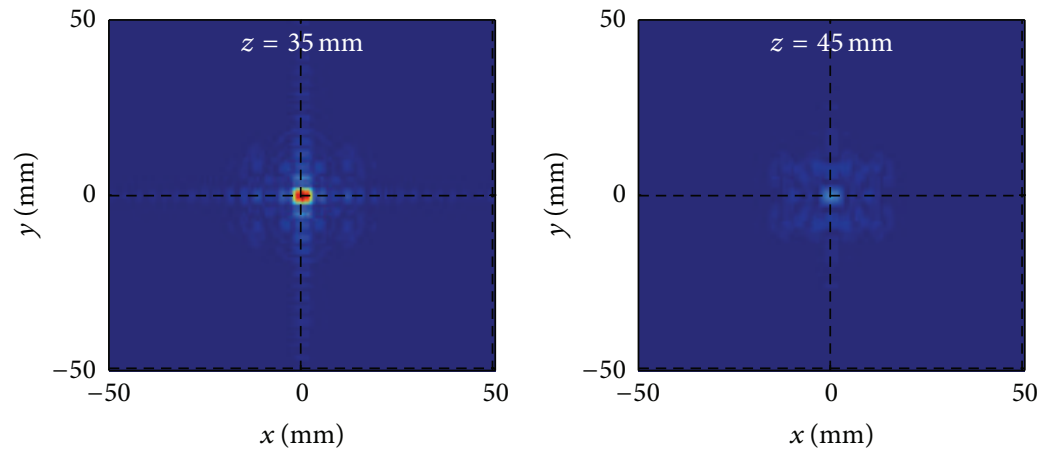

(a)

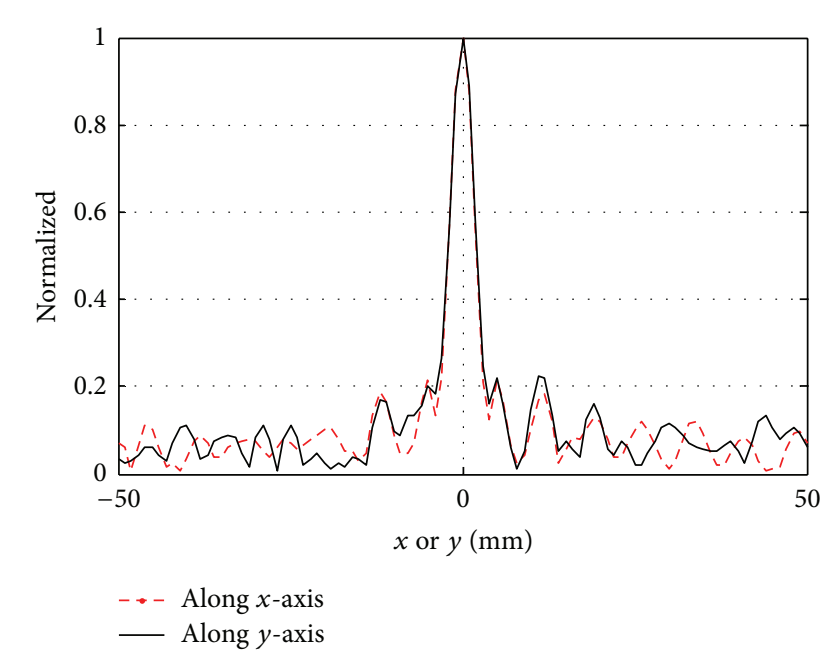

(c)

FIgURE 8: (a) Reconstructed images (shown every $10 \mathrm{~mm}$ along $z$ and only at three range locations) when the distance between the reconstruction planes is $\Delta z=5 \mathrm{~mm}$ for the example in Figure 6(a). Color bars are the same as in Figure 5. Normalized 1D magnitude of the reconstructed images along (b) range direction ( $z$ direction) and (c) cross-range directions ( $x$ and $y$ directions) in the plane of the object. The red dashed line shows the 3 -dB level below the peak (0.7 level on linear scale).

show the images every $10 \mathrm{~mm}$. The value of the Tikhonov regularization parameter $\alpha$ is $5 \times 10^{-2}$. The object is detected well at $z=60 \mathrm{~mm}$ plane (close to its true location of $z=$ $58 \mathrm{~mm}$ ) while the artifacts on the other planes are relatively low. The strongest artifact is on the plane $z=50 \mathrm{~mm}$ since it is closer to the object than the calculated range resolution limit of $17 \mathrm{~mm}$. Figure 14 shows the magnitude of the image values along the $z$ axis. It is observed again that the object is detected well at its true location. Figure 14 also shows the magnitude of the image values along the $x$ - and $y$-axes on the $z=60 \mathrm{~mm}$ plane. It is observed again that the object is detected well at its true location.

We note that here it is possible to use the waveguides in multimode operation and even below their cut-off frequency. This is because their exact incident field/Green's function is obtained through simulations and then calibrated through (29). In general, acquisition of data over a wider frequency band leads to better images. For example, Figure 15 shows the images when we use the data collected from $8.5 \mathrm{GHz}$ to $20 \mathrm{GHz}$. Although this time the lowest frequency is higher than the cut-off frequency of the $\mathrm{X}$-band waveguide $(8.2 \mathrm{GHz})$, the images suffer higher levels of artifacts.

5.4. Computational Complexity of the Inversion Technique. Here, we provide an estimate of the computational complexity of our 3D image reconstruction process. First, we denote the number of samples along $x^{\prime}$ and $y^{\prime}$ on the acquisition apertures by $N_{x^{\prime}}$ and $N_{y^{\prime}}$. The number of samples along $x$ and $y$ in each reconstructed cross-range plane is denoted by $N_{x}$ and $N_{y}$. We also denote the number of samples of $k_{x}$ and $k_{y}$ by $N_{k_{x}}$ and $N_{k_{y}}$, respectively. Table 1 summarizes the computational complexity of our approach. The flops for the FT and the inverse FT operations are provided based on the implementation of fast FT and inverse fast FT operations. The computational complexity of solving the systems of equations has been provided with the assumption that they are solved with QR factorization. The total number of flops for the image reconstruction process is the sum of all the flops in Table 1.

The execution time for the examples provided in this paper is typically less than 1 minute on a computer with Intel Xeon CPU $2.93 \mathrm{GHz}$ with $48 \mathrm{~GB}$ of RAM. 

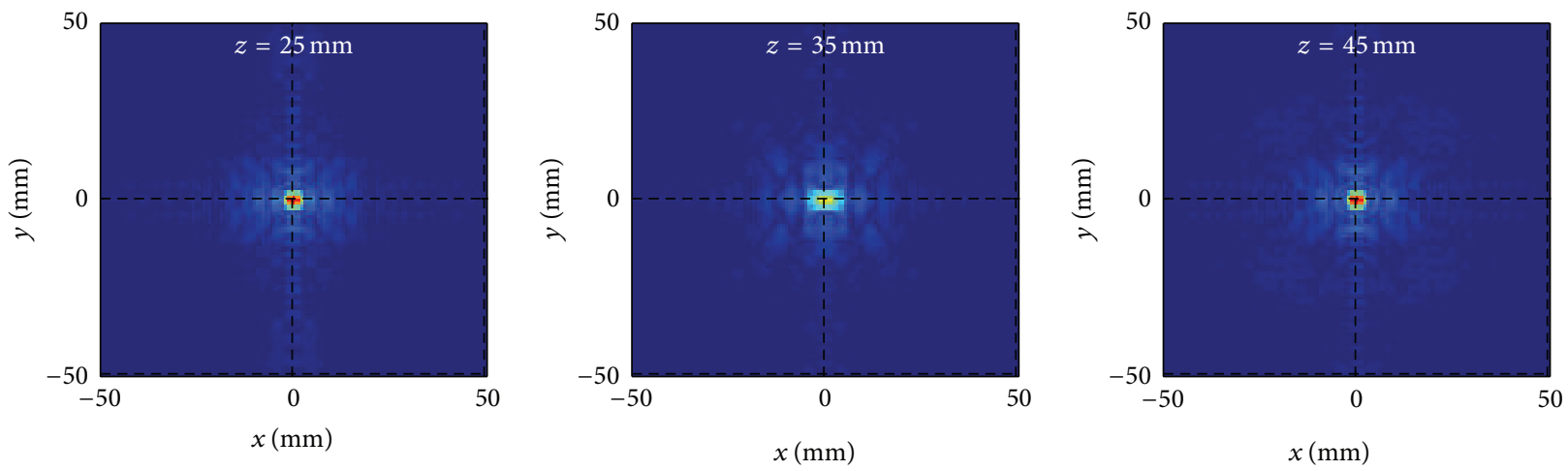

(a)
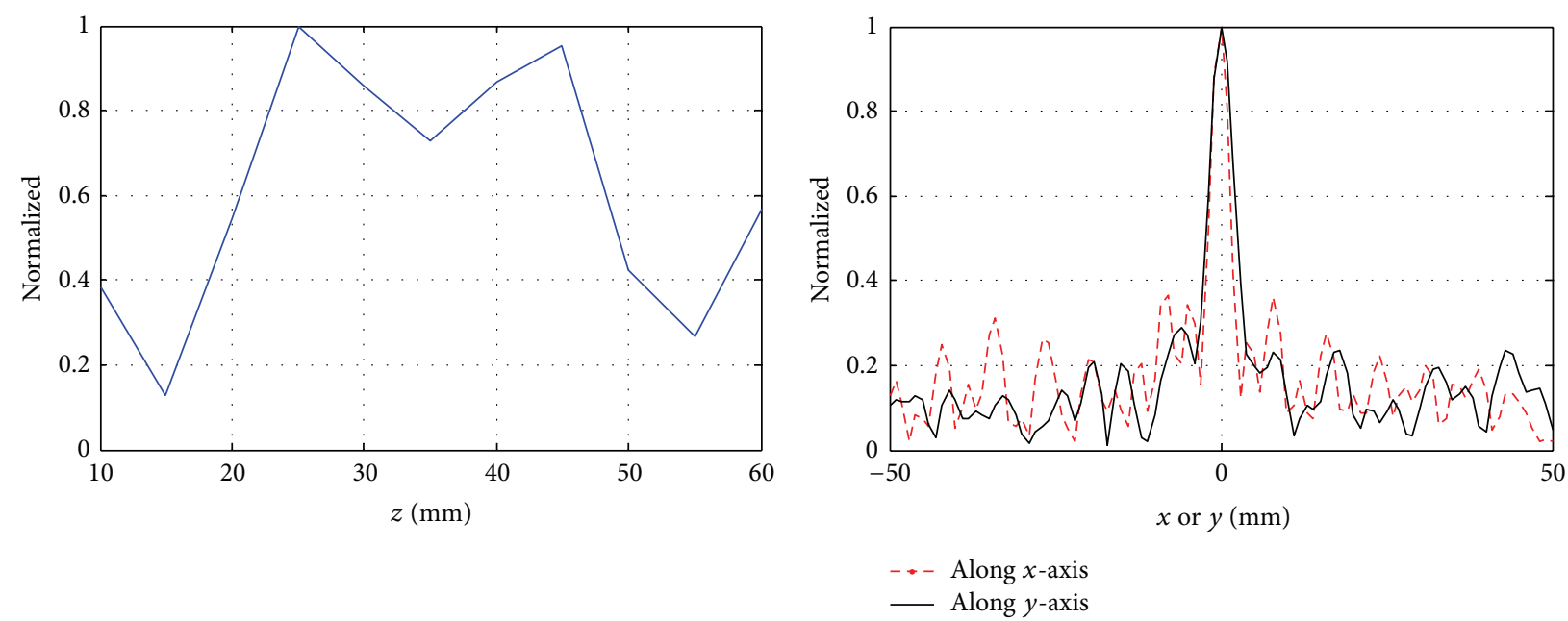

(b)

(c)

Figure 9: (a) Reconstructed images (shown every $10 \mathrm{~mm}$ along $z$ and only at three range locations) when the distance between the reconstruction planes is $\Delta z=5 \mathrm{~mm}$ for the example in Figure 6(b). Color bars are the same as in Figure 5. Normalized 1D magnitude of the reconstructed images along (b) range direction ( $z$ direction) and (c) cross-range directions ( $x$ and $y$ directions) in the plane of the object at $z=45 \mathrm{~mm}$.

\section{Conclusion and Discussion}

We proposed a multiple receiver microwave holography setup to perform 3D imaging with forward-scattered waves only that are acquired with planar raster scanning. This new setup paves the way toward microwave imaging of tissues and other materials of high loss where the back-scattered waves are too weak or are not available due to the nonreciprocal measurement system. The resolution limits for such setup are derived assuming far-field approximation.

The resolution limits proposed here are approximate values derived based on the following assumptions. (1) The background medium is homogeneous, lossless, and nondispersive. In the scenarios where the background medium is not homogenous or dispersive, these expressions can be applied with averaged parameter values for approximate estimation of the resolution limits. If the background medium is lossy, then the maximum $D_{x}$ and $D_{y}$ offset values would be limited due to the attenuation of the signal for longer paths. (2) The object is in the far zone of the antennas; that is, we only take into account the propagating waves. In nearfield imaging, however, the contribution of evanescent waves
TABLE 1: Details of computational complexity of the proposed image reconstruction process.

\begin{tabular}{ll}
\hline Operation & Number of flops \\
\hline FT of the scattered fields & $5 N_{x^{\prime}} N_{y^{\prime}} \log \left(N_{x^{\prime}} N_{y^{\prime}}\right)$ \\
$\begin{array}{l}\text { Computing functions } a_{k} \\
\text { FT of functions } a_{k}\end{array}$ & $5 N_{x} N_{y}$ \\
$\begin{array}{l}\text { Solving the systems of } \\
\text { equations in all }\end{array}$ & $N_{x} N_{y} \log \left(N_{x} N_{y}\right)$ \\
$\begin{array}{l}\text { combinations of } k_{x} \text { and } k_{y} \\
\text { Inverse FT of the contrast } \\
\text { function }\end{array}$ & $5 N_{k_{x}} N_{k_{y}}\left(10 N_{\omega} N_{z}^{2}+10 N_{\omega} N_{z}+N_{z}^{2}\right)$ \\
\hline
\end{tabular}

leads to better resolution beyond what is computed from the expressions derived here.

Through simulation results, we showed that for dimensions typically used in microwave imaging, the spatial resolution limits provide acceptable image quality. The achieved range and cross-range resolution limits for the proposed setup are comparable or below a centimeter in the frequency range from $3 \mathrm{GHz}$ to $10 \mathrm{GHz}$. 

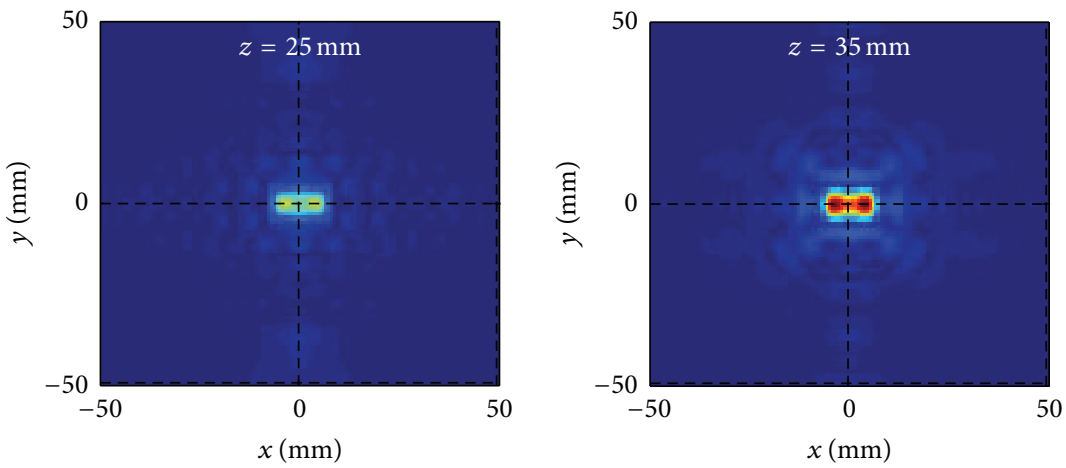

(a)

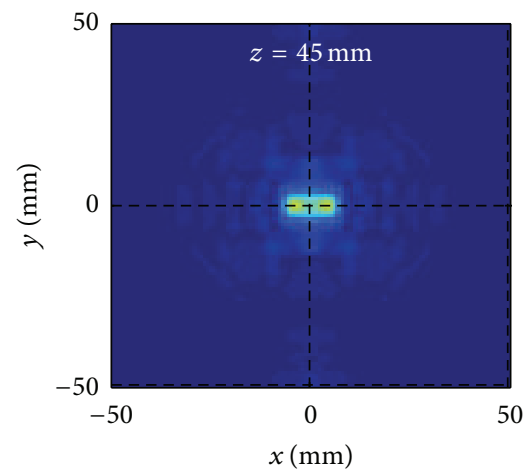

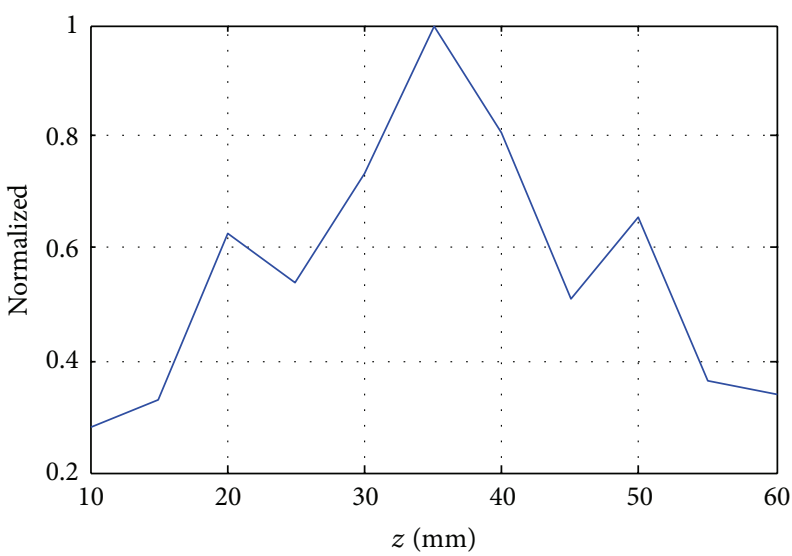

(b)

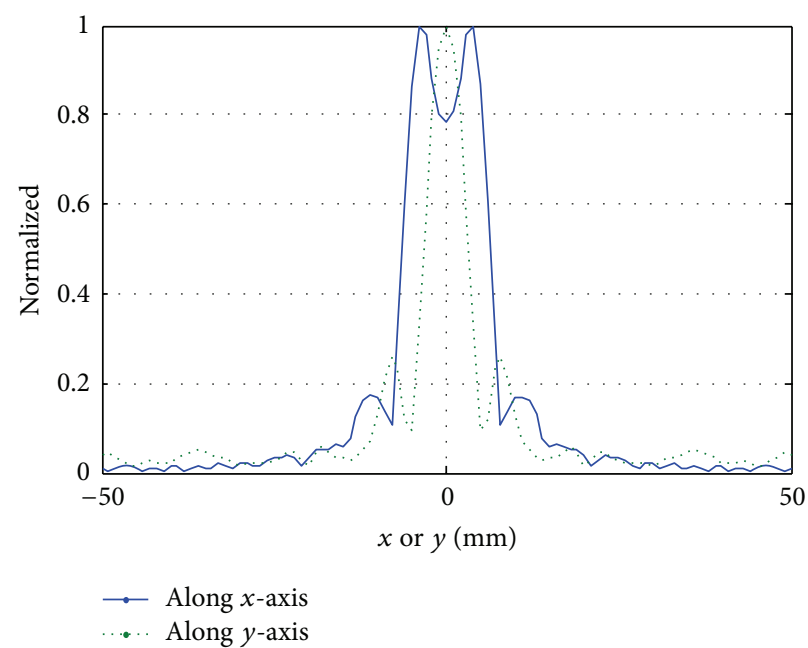

(c)

Figure 10: (a) Reconstructed images (shown every $10 \mathrm{~mm}$ along $z$ and only at three range locations) when the distance between the reconstruction planes is $\Delta z=5 \mathrm{~mm}$ for the example in Figure 6(c). Color bars are the same as in Figure 5. Normalized 1D magnitude of the reconstructed images along (b) range direction ( $z$ direction) and (c) cross-range directions ( $x$ and $y$ directions) in the plane of the objects.

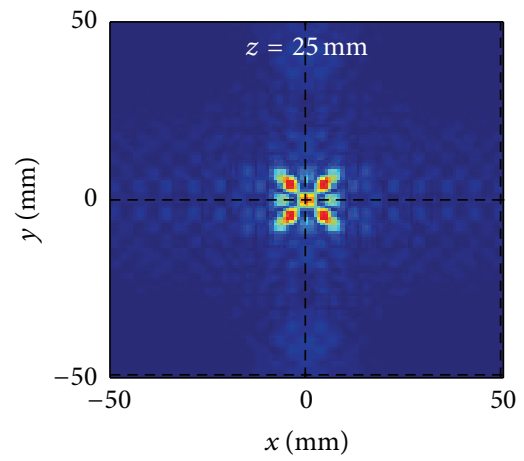

(a)

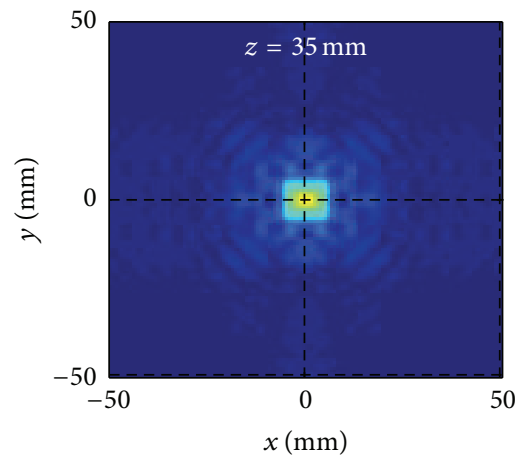

(b)

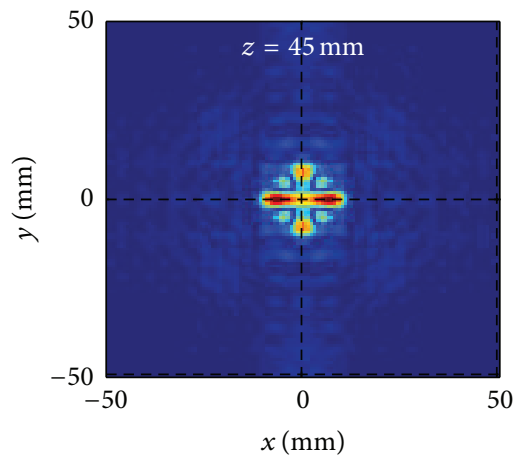

(c)

FIGURE 11: Reconstructed images (shown every $10 \mathrm{~mm}$ along $z$ and only at three range locations) when the distance between the reconstruction planes is $\Delta z=5 \mathrm{~mm}$ for the example in Figure 6(d). Color bars are the same as those in Figure 5.

We also evaluated the performance of the proposed 3D imaging method via an experiment in free space with a 5-element array of X-band open-ended waveguides. The satisfactory results justify further experimental work toward tissue imaging with microwave holography where the sensors must have significantly reduced size and improved sensitivity. In tissue imaging, the SNR values can vary significantly based on (1) the utilized antennas, (2) the acquisition setup and measurement instrumentation, and (3) the properties of the tissue. All of these factors would affect the choice of 


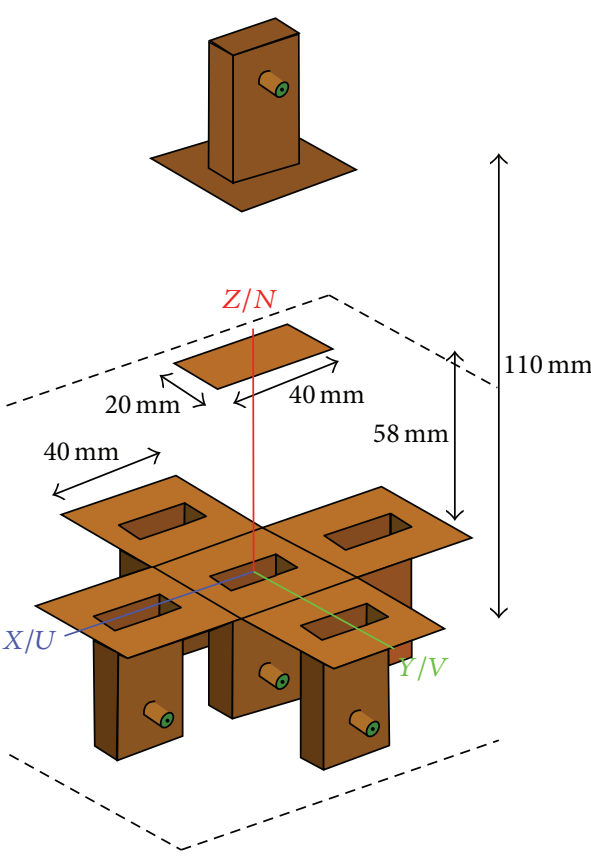

(a)

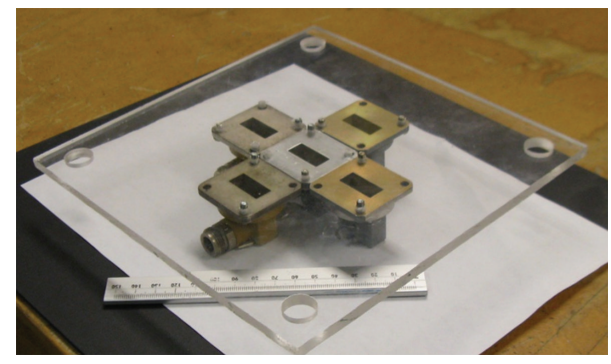

(b)

FIGURE 12: (a) Illustration of the experimental imaging setup consisting of six X-band open-ended waveguides where one acts as a transmitter and five act as receivers. A rectangular metallic sheet is being imaged. (b) Image of five open-ended waveguides attached together to construct the multiple receiver array.
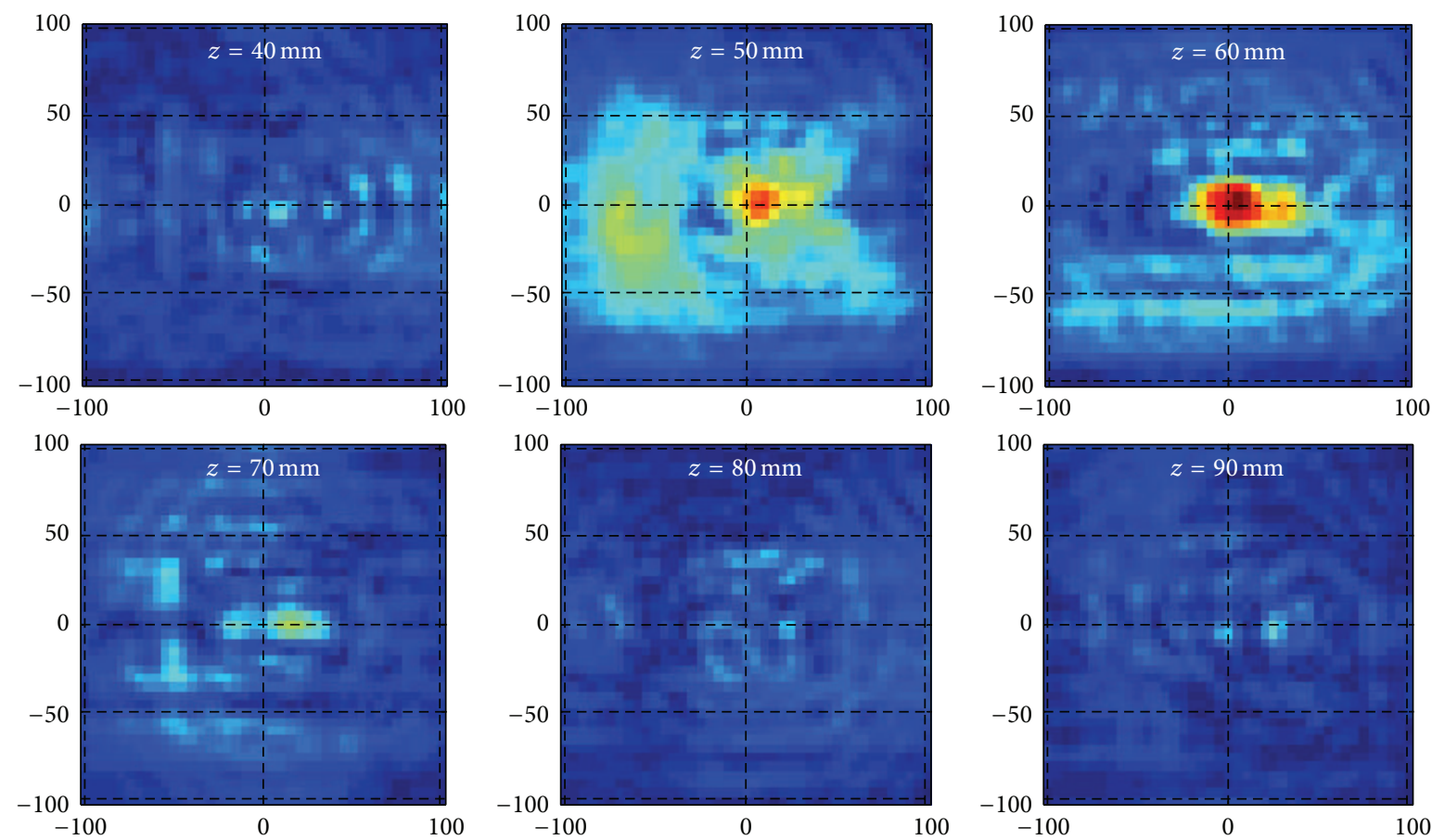

FIGURE 13: Reconstructed images (shown every $10 \mathrm{~mm}$ along the $z$ axis) when the distance between the reconstruction planes is $\Delta z=5 \mathrm{~mm}$ using the data in the frequency range from $3 \mathrm{GHz}$ to $20 \mathrm{GHz}$ in the experimental example. Color bars are the same as those in Figure 5 . The horizontal and vertical axes show the positions along the $x$ and $y$ directions in terms of $\mathrm{mm}$. 


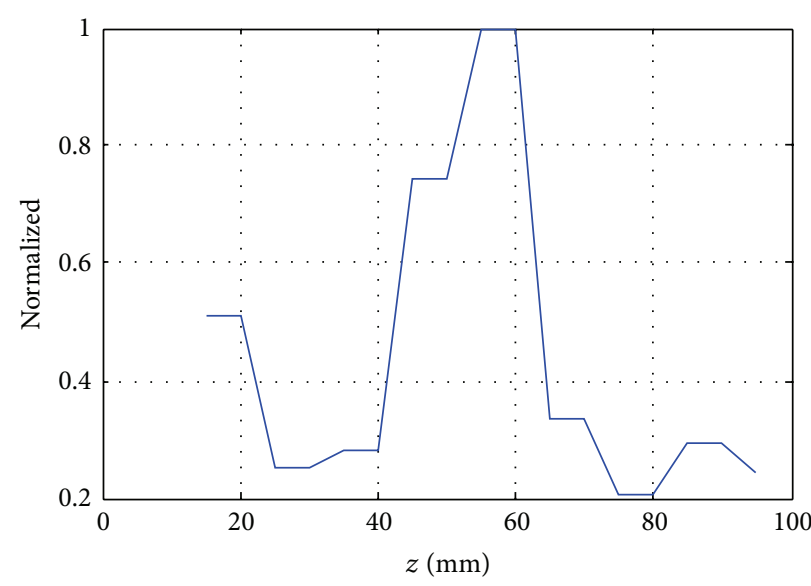

(a)

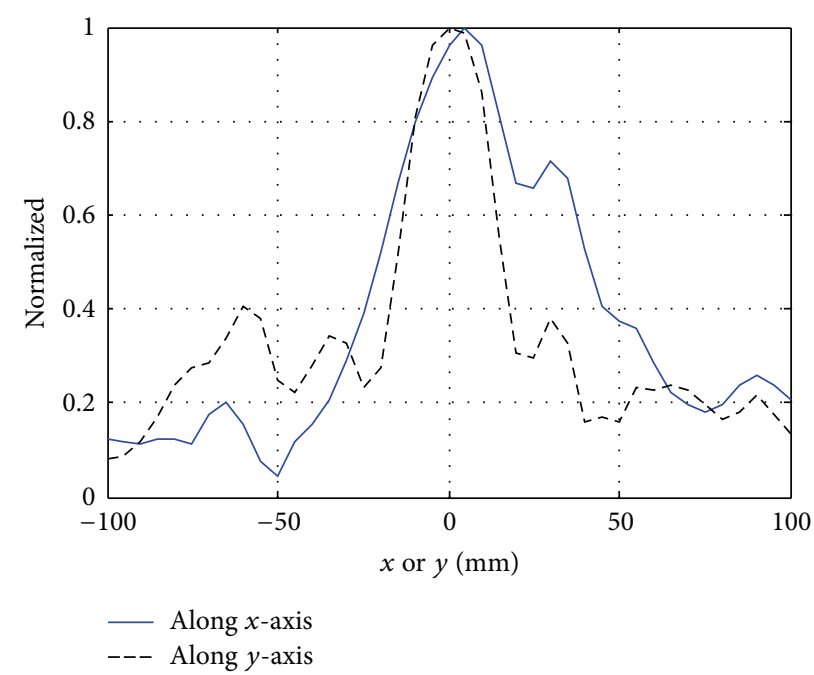

(b)

FIGURE 14: Normalized 1D magnitude of the reconstructed images along the range direction ( $z$ direction) and cross-range directions ( $x$ and $y$ directions) on the plane of the object in the experiment.
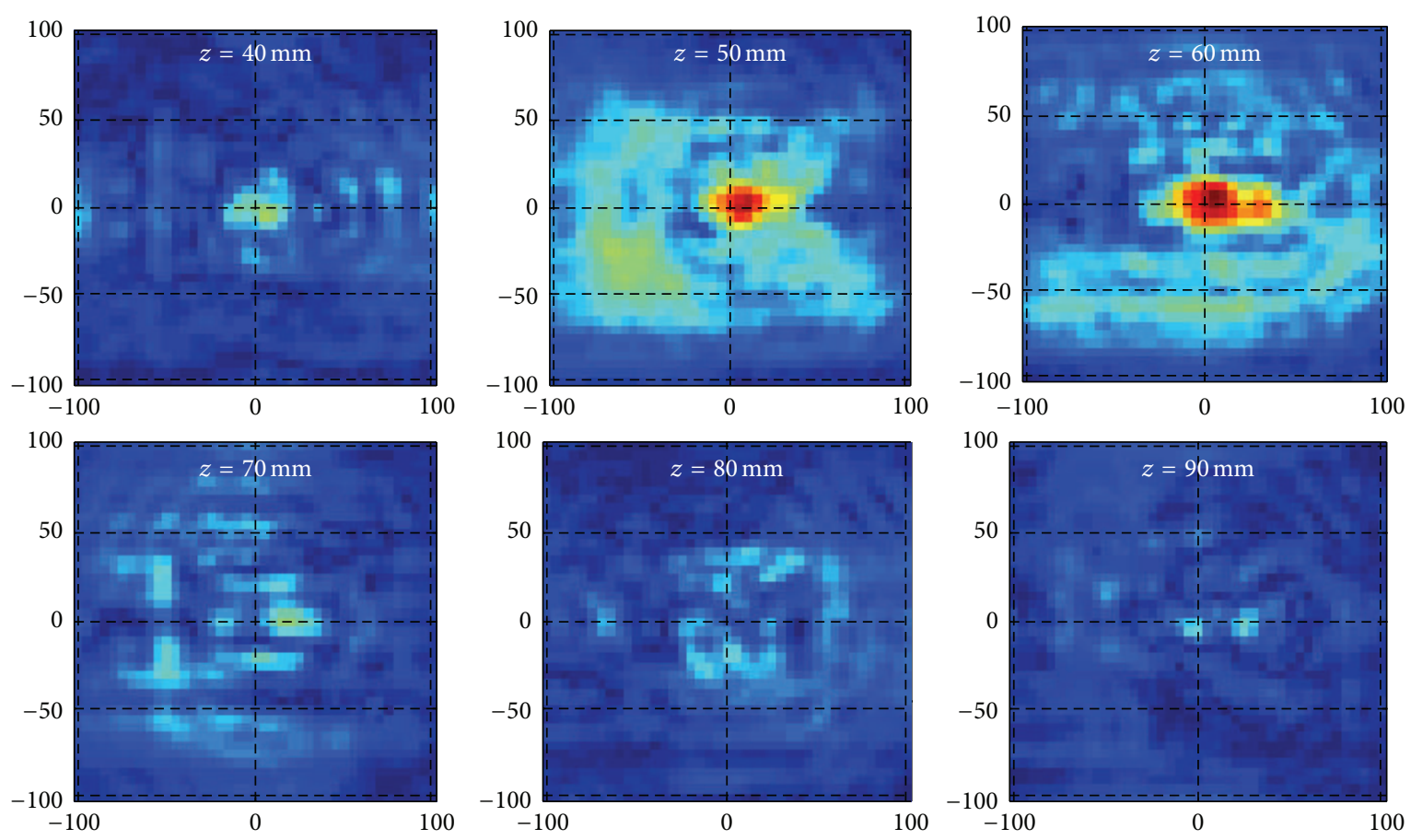

FIGURE 15: Reconstructed images (shown every $10 \mathrm{~mm}$ along $z$ ) when the distance between the reconstruction planes is $\Delta z=5 \mathrm{~mm}$ using the data in the frequency range from $8.5 \mathrm{GHz}$ to $20 \mathrm{GHz}$ in the experimental example. Color bars are the same as those in Figure 5. The horizontal and vertical axes show the positions along the $x$ and $y$ directions in terms of $\mathrm{mm}$.

the proper $\Delta z$ value as well as the sensitivity of the system. However, these can be determined empirically using simple phantom measurements with the particular hardware setup.

Although the objects are symmetric in the cross-range plane, the reconstructed images are slightly asymmetric. The first factor which affects both the simulation and measurement results is the noise in the scattered field. The simulated scattered field suffers from the numerical noise as discussed in Section 5 while the measured scattered field suffers from mechanical positioning errors during raster scanning, from electronic noise in the measurement equipment and from electromagnetic interference from the environment. In the case of the experimental results, the slight errors in the numerical evaluation of the kernel in (20) intensify this issue further.

As we discussed earlier, increasing the offset values $D_{x}$ and $D_{y}$ leads to improvement in the resolution. However, in lossy background media the offsets can be increased as 
much as the received signals remain reliable. The optimal offsets are determined based on the amount of loss in the background medium, size and contrast of the objects to be detected, radiation pattern of the antennas, and the maximum allowable dimension of the imaging setup. Thus, the optimal offset values would be highly casedependent.

We reiterate that the proposed reconstruction method is based on the linear Born approximation with the entailing limitations. The inclusion of electrically large objects in the imaged region will inevitably lead to artifacts in the reconstructed images. Yet, the large object and other possible inclusions in this object will be detected as demonstrated by a simulation example. In the experimental example, the object is again a large copper sheet and our algorithm is again capable of producing satisfactory images where the object is clearly visible. In general, it is well known that the accuracy of the imaging results with methods based on the Born approximation degrades for high-contrast and large objects, the degradation usually being in the form of increased image artifacts. On the other hand, the algorithms which are not based on the Born approximation (e.g., modelbased optimization approaches) suffer from ill-conditioning especially in $3 \mathrm{D}$ imaging. The ill-conditioning also makes them prone to errors in the forward models they use, which may be significant in full-wave simulation models. Such algorithms also suffer from nonuniqueness of the solution and often fail to converge to a meaningful solution. From a practical standpoint, such algorithms are also often in a disadvantage because they require extensive computational time and memory. Our proposed 3D imaging is fast, does not require a full-wave forward model, and is significantly less illconditioned. Future work is now focused on an approach to tissue imaging, which builds on the result of the presented holography method and overcomes the limitations of the Born approximation.

\section{References}

[1] N. K. Nikolova, "Microwave imaging for breast cancer," IEEE Microwave Magazine, vol. 12, no. 7, pp. 78-94, 2011.

[2] N. H. Farhat and W. R. Guard, "Millimeter wave holographic imaging of concealed weapons," Proceedings of the IEEE, vol. 59, no. 9, pp. 1383-1384, 1971.

[3] E. J. Baranoski, “Through-wall imaging: historical perspective and future directions," Journal of the Franklin Institute, vol. 345, no. 6, pp. 556-569, 2008.

[4] R. Zoughi, Microwave Non-Destructive Testing and Evaluation, Kluwer Academic Publishers, 2000.

[5] M. Pastorino, Microwave Imaging, Wiley, Hoboken, NJ, USA, 2010.

[6] N. H. Farhat, "Microwave holography and its applications in modern aviation," in Engineering Applications of Holography Symposium, Proceedings of SPIE, pp. 295-314, 1972.

[7] G. Tricoles and N. H. Farhat, "Microwave holography: applications and techniques," Proceedings of the IEEE, vol. 65, no. 1, pp. 108-121, 1977.

[8] B. P. Hildebrand and K. A. Haines, "Holography by scanning," The Journal of the Optical Society of America, vol. 59, no. 1, pp. $1-6,1969$.
[9] B. P. Hildebrand and B. B. Brenden, An Introduction to Acoustical Holography, Plenum, New York, NY, USA, 1972.

[10] D. Gabor, "A new microscopic principle," Nature, vol. 161, no. 4098, pp. 777-778, 1948.

[11] D. M. Sheen, D. L. McMakin, and T. E. Hall, "Three-dimensional millimeter-wave imaging for concealed weapon detection," IEEE Transactions on Microwave Theory and Techniques, vol. 49, no. 9, pp. 1581-1592, 2001.

[12] M. Soumekh, "Bistatic synthetic aperture radar inversion with application in dynamic object imaging," IEEE Transactions on Signal Processing, vol. 39, no. 9, pp. 2044-2055, 1991.

[13] M. Soumekh, "A system model and inversion for synthetic aperture radar imaging," IEEE Transactions of Image Processing, vol. 1, no. 1, pp. 64-76, 1992.

[14] M. Soumekh, Fourier Array Imaging, Prentice-Hall, Englewood Cliffs, NJ, USA, 1994.

[15] D. C. Munson Jr., J. D. O’Brien, and W. K. Jenkins, "A tomographic formulation of spotlight-mode synthetic aperture radar," Proceedings of the IEEE, vol. 17, no. 8, pp. 917-925, 1983.

[16] D. Sheen, D. McMakin, and T. Hall, "Near-field threedimensional radar imaging techniques and applications," Applied Optics, vol. 49, no. 19, pp. E83-E93, 2010.

[17] A. Dallinger, S. Schelkshorn, and J. Detlefsen, "Efficient $\omega$ $\mathrm{k}$-algorithm for circular SAR and cylindrical reconstruction areas," Advances in Radio Science, vol. 4, pp. 85-91, 2006.

[18] W. X. Tan, W. Hong, Y. P. Wang, and Y. R. Wu, "A novel spherical-wave three-dimensional imaging algorithm for microwave cylindrical scanning geometries," Progress in Electromagnetics Research, vol. 111, pp. 43-70, 2011.

[19] R. K. Amineh, M. Ravan, A. Khalatpour, and N. K. Nikolova, "Three-dimensional near-field microwave holography using reflected and transmitted signals," IEEE Transactions on Antennas and Propagation, vol. 59, no. 12, pp. 4777-4789, 2011.

[20] R. K. Amineh, A. Khalatpour, H. Xu, Y. Baskharoun, and N. Nikolova, "Three-dimensional near-field microwave holography for tissue imaging," International Journal of Biomedical Imaging, vol. 2012, Article ID 291494, 11 pages, 2012.

[21] A. Li, "Algorithms for the implementation of Stolt interpolation in SAR processing," in Proceedings of the IEEE International Geoscience and Remote Sensing Symposium, vol. 1, pp. 360-362, 1992.

[22] B. Subiza, E. Gimeno-Nieves, J. M. Lopez-Sanchez, and J. Fortuny-Guasch, "An approach to SAR imaging by means of non-uniform FFT'," in Proceedings of the IEEE International Geoscience and Remote Sensing Symposium, vol. 6, pp. 40894091, July 2003.

[23] R. K. Amineh, M. Ravan, A. Trehan, and N. K. Nikolova, "Nearfield microwave imaging based on aperture raster scanning with TEM horn antennas," IEEE Transactions on Antennas and Propagation, vol. 59, no. 3, pp. 928-940, 2011.

[24] I. Walterscheid, A. R. Brenner, and J. H. G. Ender, "Results on bistatic synthetic aperture radar," Electronics Letters, vol. 40, no. 19, pp. 1224-1225, 2004.

[25] A. N. Tikhonov and V. Y. Arsenin, Solutions of Ill-Posed Problems, Wiley, New York, NY, USA, 1977.

[26] http://www.mathworks.com/matlabcentral/fileexchange/52regtools.

[27] EM softwares \& systems-S.A. (Pty) Ltd., http://www.feko.info/.

[28] D. R. Wehner, High-Resolution Radar, Artech House, 1995. 

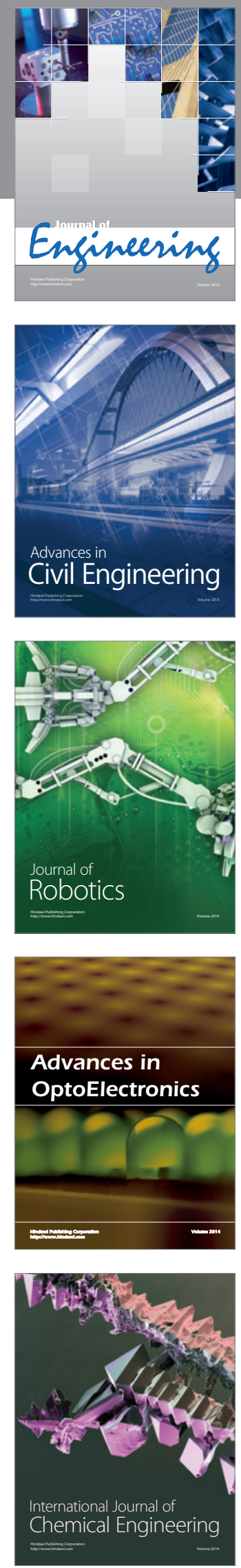

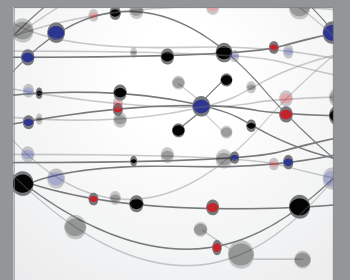

The Scientific World Journal
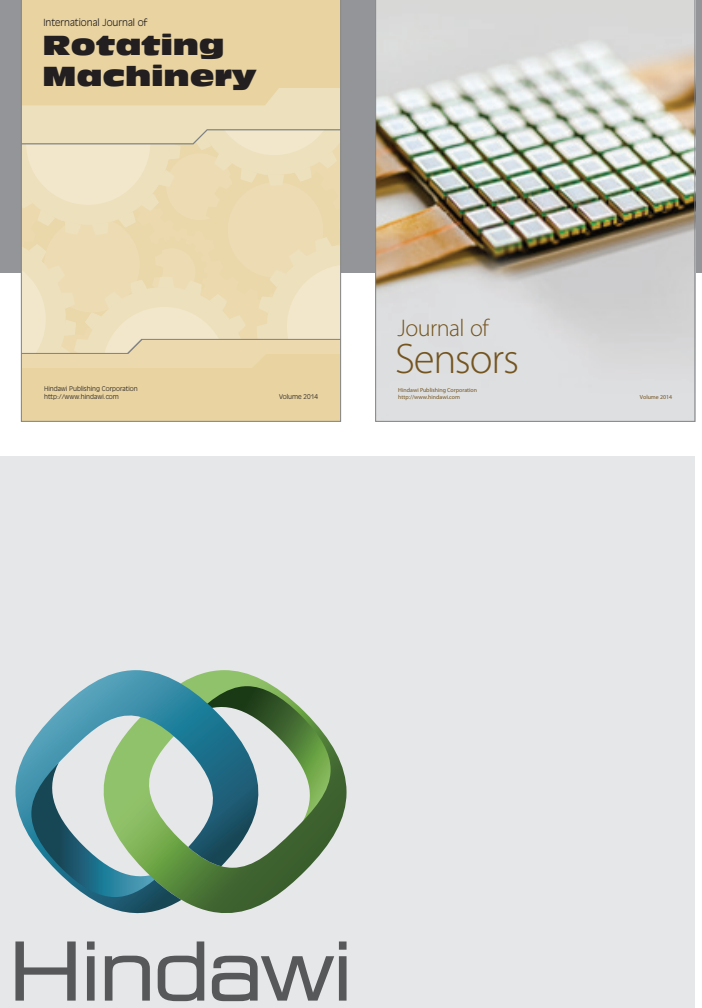

Submit your manuscripts at http://www.hindawi.com
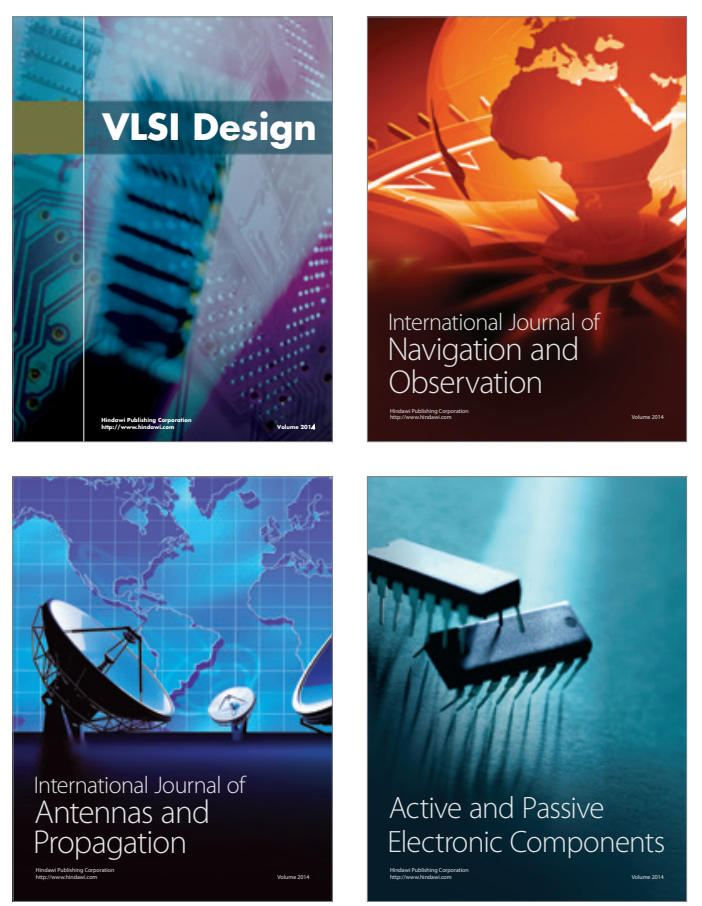
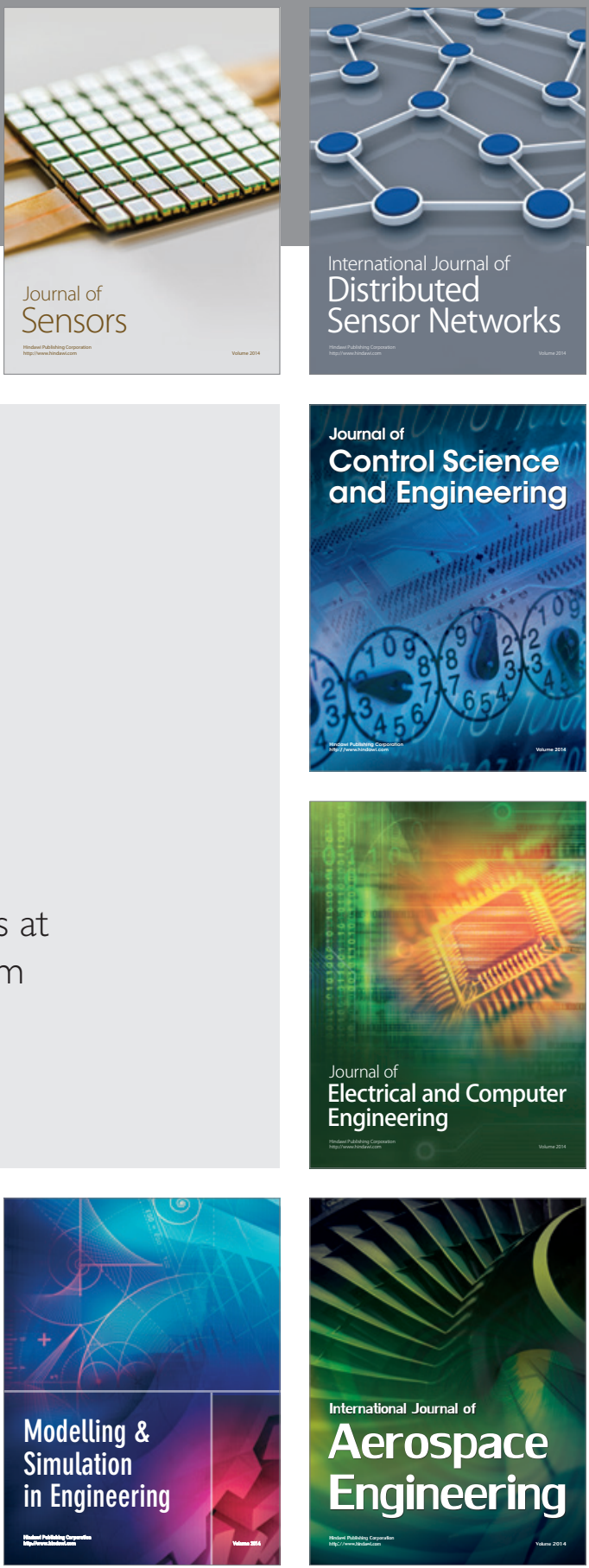

Journal of

Control Science

and Engineering
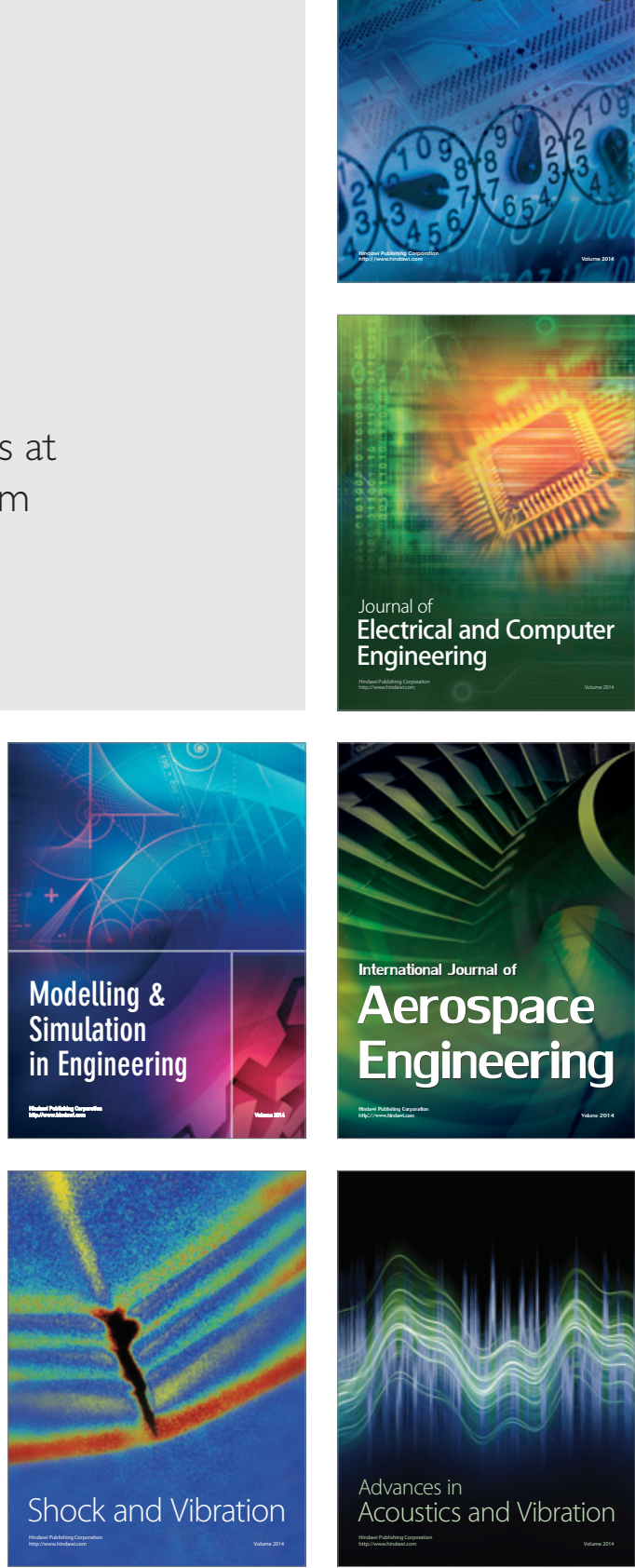